\title{
Vertex opposition in spherical buildings
}

\author{
Anna Kasikova and Hendrik Van Maldeghem
}

\begin{abstract}
We study to which extent all pairs of opposite vertices of self-opposite type determine a given building. We provide complete answers in the case of buildings related to projective spaces, to polar spaces and the exceptional buildings, but for the latter we restrict to the vertices whose Grassmannian defines a parapolar space of point diameter 3. Some results about non-self opposite types for buildings of types $\mathrm{A}_{n}, \mathrm{D}_{m}(m$ odd $)$, and $\mathrm{E}_{6}$ are also provided.
\end{abstract}

\section{Introduction}

In this paper, the main aim is essentially to prove that the opposition relation in a spherical building $\mathcal{B}$ restricted to one self-opposite type of vertices uniquely determines $\mathcal{B}$. However, this is not true in full generality, as for instance, there are known counterexamples for generalized hexagons, see [5], and we will discover a second class of counterexamples (of unbounded rank) in this paper.

Given a particular building $\mathcal{B}$ and a particular self-opposite type $t$ of vertices, then the problem of reconstructing $\mathcal{B}$ from all opposite pairs of vertices of type $t$, is usually not a too hard exercise, especially when $\mathcal{B}$ is finite (elementary counting techniques allow to recognize the different vertices of $\mathcal{B}$ ) or of classical type. However, if it is only known that $\mathcal{B}$ belongs to a class of buildings, and $t$ belongs to a set of self-opposite types, the question becomes much harder, and more interesting. Also, the implications become stronger, as this implies that the structures of opposition restricted to vertices of given types differ whenever the buildings or the types differ. And we also discover cases in which these structures coincide for different buildings.

The ultimate problem is to consider all spherical buildings and all self-opposite types. In this paper, however, we have restricted our attention to all projective and polar spaces, and to the popular point-line geometries arising from the (mainly exceptional) spherical buildings, i.e., we restrict the type of vertices to those types whose Grassmannians define parapolar spaces of diameter 3. This includes (with Bourbaki labeling) types 1 and 4 for buildings of type $F_{4}$, type 2 for buidings of type $E_{6}$, types 1 and 7 for buildings of type $\mathrm{E}_{7}$, and type 8 for buildings of type $\mathrm{E}_{8}$. The overlap with polar spaces consists of the line Grassmannian of polar spaces themselves (type 1 for buildings of types $\mathrm{B}_{n}$ and $\mathrm{D}_{n}$ ). Informally, we can state our main result as follows: 
Main Result 1 - Informal statement. Let $\mathcal{B}$ be a building related to a projective space or a polar space (this polar space must have thick lines but can be non-thick otherwise-correspondingly, $\mathcal{B}$ can be non-thick of type $\mathrm{B}_{n}$ or thick of type $\mathrm{D}_{n}$ ), except that we do not allow $\mathcal{B}$ to correspond to a non-degenerate quadratic form of maximal Witt index $2 n-1$ in $(4 n-2)$-dimensional projective spaces, or let $\mathcal{B}$ be a building of exceptional type. Let $t$ be a self-opposite type in $\mathcal{B}$, with the restriction that the $t$-Grassmannian defines a parapolar space of diameter 3 if $\mathcal{B}$ is of exceptional type. Then the set of all opposite pairs of vertices of type $t$ unambiguously determines $\mathcal{B}$.

Concerning the parapolar spaces of diameter 3, there are essentially five different mutually positions for two points: they can coincide (CASE 0), they can be collinear (CASE 1), they can be at distance 2 in the collinearity graph, with at least two common neighbours (CASE 2), they can be at distance 2 in the collinearity graph, with exactly one common neighbour (CASE 3), or they can be opposite (and then they have distance 3 in the collinearity graph of the parapolar space, CASE 4). Our second main result slightly extends the first one for these parapolar spaces.

Main Result 2 - Informal statement. Let $\Gamma$ be a parapolar space of diameter 3 arising from a spherical building $\mathcal{B}$. Let $i$ be such that $1<i \leq 4$. Then the set of pairs of points of $\Gamma$ in mutual positions $C A S E j$, with $j \geq i$, uniquely determines $\Gamma$, and hence the corresponding building $\mathcal{B}$.

This statement is trivial for $i=2$. For $i=3$, it is proved with not much additional effort together with the case $i=4$, which is part of Main Result 1. And that is exactly why we also include it.

For precise statements, we refer to the subsequent sections.

In order to prove our main results, we introduce the concepts of a round-up triple, and of a geometric line. These notions can be studied on their own right, and they appear to have tight connections with (minimal) projective embeddings of the corresponding Grassmann geometry. In the course of our proof, we determine all round-up-triples and all geometric lines of Grassmann geometries corresponding with an arbitrary type in any polar space, and of parapolar spaces related to spherical buildings.

The paper is organized as follows. In the next section, we define the geometries that are under consideration in this paper. In Section 3, we introduce the notion of round-up triple, and prove some preliminary statements. We have chosen to do this in full generality in the framework of arbitrary spherical buildings. However, we will not define all notions that we need there (for example, the precise definition of a building, retraction onto apartments, etc.) but refer to the literature such as $[1,10,12]$. In Section 4 , we prove our main result for polar spaces. Section 5 deals with strong parapolar spaces, i.e., opposition of vertices of type 7 in buildings of type $\mathrm{E}_{7}$ and vertices of type $n$ in buildings of type $\mathrm{A}_{2 n-1}$. Here, we also prove an analogue of our main results for vertices of type 1 and 6 in buildings of type $\mathrm{E}_{6}$, and for vertices of arbitrary type in projective paces. Section 6 finally deals with the remaining parapolar spaces of point diameter 3 . . 


\section{Point-line definitions}

Our definitions mostly follow [11].

A point-line space $(\mathcal{P}, \mathcal{L})$ is a set $\mathcal{P}$ together with a set $\mathcal{L}$ of subsets of $\mathcal{P}$, such that each $L \in \mathcal{L}$ has at least two elements. Point-line spaces can be identified with point-line geometries (that is, bipartite graphs with the two parts labeled "points" and "lines") satisfying two requirements: no two lines are incident with exactly the same sets of points and each line is incident with at least two points. The point-collinearity graph of a pointline space $\Gamma=(\mathcal{P}, \mathcal{L})$ is a graph with vertex set $\mathcal{P}$ in which two points $p, q \in \mathcal{P}$ are adjacent if and only if they are distinct collinear points of $\Gamma$. A point-line space is connected if its point-collinearity graph is connected. For a point $p$ of a point-line space $\Gamma$ we denote $p^{\perp}$ the set of all points of $\Gamma$ collinear with $p$; the set $p^{\perp}$ includes the point $p$ itself. A line is called thick if it has at least three points. The point-line space $(\mathcal{P}, \mathcal{L})$ is said to have thick lines if all members of $\mathcal{L}$ are thick.

A partial linear space is a point-line space in which every pair of distinct points $x, y$ is contained in at most one line, denoted by $\langle x, y\rangle$.

A set $S \subseteq \mathcal{P}$ is called a subspace if, whenever two points of a line $L$ belong to $S$, then all points of $L$ belong to $S$. A singular subspace is a subspace that corresponds to a clique in the point-collinearity graph. A subspace is called convex if, whenever two points $p, q$ belong to it, then so do all points on any shortest path from $p$ to $q$ in the point-collinearity graph of $(\mathcal{P}, \mathcal{L})$.

We say here that a subspace of a point-line space $\Gamma$ is a geometric hyperplane of $\Gamma$ if it is a proper subspace meeting every line nontrivially.

A gamma space is a point-line space satisfying one additionall axiom - for every nonincident point-line pair $(p, L)$, if the point $p$ is collinear with at least two distinct points of $L$, then $p$ is collinear with all points of $L$.

The gamma space $(\mathcal{P}, \mathcal{L})$ is a polar space if $(1)$ every line has at least three points, if $(2)$ the length of nested sequences of singular subspaces is bounded, and if (3) for every point $p \in \mathcal{P}, p^{\perp}$ is a geometric hyperplane. In a polar space, the singular subspaces endowed with the lines contained in it are projective spaces. By definition, there is an $r \in \mathbb{N}$ such that some singular subspace has dimension $r-1$, and no singular subspace has dimension exceeding $r-1$. Then $r$ is called the rank of the polar space. Here, any line is considered as a projective line. For convenience, we view a set of cardinality at least 2 without lines as a polar space of rank 1, and every element of the set is viewed as a projective space of dimension 0. A gamma space only satisfying (1) and (3), but not (2) is a polar space of infinite rank.

Polar spaces of rank 2 are generalized quadrangles, but the converse is only true if one requires that the generalized quadrangle has thick lines, i.e., every line contains at least three points. Hence, a thick generalized quadrangle is a polar space of rank 2 which is also a dual polar space. In general, a generalized quadrangle is either a grid (including $2 \times k$ grids, with $k>1$ any cardinal), a dual grid, a polar space of rank 2 , or the dual of such. 
Suppose $\Gamma$ is a point-line space. A symplecton of $\Gamma$ is a convex subspace $S$ of $\Gamma$ such that the point-line space induced by $\Gamma$ on $S$ is a polar space of rank at least 3, or a generalized quadrangle.

Suppose $\Delta=(V, E)$ is a graph. Let $k \geq 0$ be an integer. Let $u, v \in V$. Then we denote $\mathrm{d}_{\Delta}(u, v)$ the length of a shortest possible walk from $u$ to $v$ in $\Delta$, and we call this number the distance from $u$ to $v$ in $\Delta$. We let $\Delta_{k}(u)=\left\{v \in V \mid \mathrm{d}_{\Delta}(u, v)=k\right\}$ and we let $\Delta_{k}^{*}(u)=\left\{v \in V \mid \mathrm{d}_{\Delta}(u, v) \leq k\right\}$.

Supppose $\Gamma=(\mathcal{P}, \mathcal{L})$ is a point-line space with point-collinearity graph $\Delta$. Then $\Gamma$ is a parapolar space if it satisfies the following axioms.

(PPS1) $\Gamma$ is connected and is a gamma space.

(PPS2) For every pair $p, q \in \mathcal{P}$ with $\mathrm{d}_{\Delta}(p, q)=2$, either $\left|p^{\perp} \cap q^{\perp}\right|=1$ or $\{p, q\}$ is contained in a symplecton.

(PPS3) Every line of $\Gamma$ is contained in a symplecton.

Let $\Gamma=(\mathcal{P}, \mathcal{L})$ be a parapolar space with point-collinearity graph $\Delta$. We say that $\Gamma$ has symplectic rank at least $r$ if every symplecton of $\Gamma$ is a polar space of (polar) rank at least $r$. Suppose $p, q \in \mathcal{P}$ are such that $\mathrm{d}_{\Delta}(p, q)=2$. If $\left|p^{\perp} \cap q^{\perp}\right|=1$ then we say that $\{p, q\}$ is a special pair; if $\{p, q\}$ is contained in a symplecton, then $\{p, q\}$ is a symplectic pair and we denote $\mathrm{S}(p, q)$ the unique symplecton of $\Gamma$ containing $\{p, q\}$. We denote $\mathcal{S}$ the set of all symplecta of $\Gamma$. For a point $p \in \mathcal{P}$, we let

$$
\begin{gathered}
\Delta_{\text {symp }}(p)=\left\{p^{\prime} \in \Delta_{2}(p) \mid\left\{p, p^{\prime}\right\} \text { is a symplectic pair }\right\} \\
\Delta_{\text {spec }}(p)=\left\{p^{\prime} \in \Delta_{2}(p) \mid\left\{p, p^{\prime}\right\} \text { is a special pair }\right\}
\end{gathered}
$$

A parapolar space is a strong parapolar space if it has no special pairs.

Let $\Gamma=(\mathcal{P}, \mathcal{L})$ be a point-line space and suppose $\Delta$ denotes the point-collinearity graph of $\Gamma$. For a point $p \in \mathcal{P}$, we denote $\Gamma_{p}$ the geometry of lines and projective planes of $\Gamma$ on $p$. We call $\Gamma_{p}$ the point residue of $\Gamma$ at $p$ and we denote $\Delta_{p}$ the point-collinearity graph of $\Gamma_{p}$.

If the parapolar space $\Gamma$ with diameter 3 arises from a spherical building $\mathcal{B}$, then its points are the vertices of $\mathcal{B}$ of some distinguished type, and if that type is self-opposite, then opposite vertices correspond with points at maximal distance in the point-collinearity graph.

In a polar space $(\mathcal{P}, \mathcal{L})$, two singular subspaces $U, V$ are opposite if no point of $U \cup V$ is collinear with all points of $U \cup V$. Opposite singular subspaces necessarily have the same dimension. The complex formed by all singular subspaces, structured by inclusion, naturally forms a spherical building, in which the opposition relation for vertices coincides with the opposition relation just defined.

The polar space $(\mathcal{P}, \mathcal{L})$ of rank $r$ is non-thick if every next-to-maximal singular subspace is contained in exactly two maximal subspaces (the latter are also called generators). In this case, the maximal subspaces fall into two classes, where two generators are in the same class if and only their intersection has even codimension in both. The oriflamme complex 
is the geometry consisting of all singular subspaces, except for the next-to-maximal ones, where the two classes of generators are different types of elements of the complex, two of these being incident (or connected, or forming a simplex) if they intersect in a next-tomaximal subspace. This complex defines a building of type $\mathrm{D}_{r}, r \geq 2$. Here, the types corresponding to the generators are self-opposite if and only if $r$ is even (in general, a type is called self-opposite if there exist two opposite elements of that type).

\section{Round-up triples}

In this section we introduce the notion of round-up triple, first in a very general setting, and then specializing to geometries and buildings.

\subsection{Generalities}

Suppose $\mathcal{P}$ is a set and $\mathcal{F}$ is a set of subsets of $\mathcal{P}$. For a set $T \subseteq \mathcal{P}$ of size 3 , we say that $T$ is an $\mathcal{F}$-round-up triple of $\mathcal{P}$ if no element of $\mathcal{F}$ intersects $X$ at exactly two points. That is, for every $X \in \mathcal{F},|X \cap T| \geq 2$ implies $T \subseteq X$. If we let $\mathcal{L}$ to be a set of $\mathcal{F}$-round-up triples, then every element of $\mathcal{F}$ becomes a subspace of the point-line space $(\mathcal{P}, \mathcal{L}$ ) (which might not be a partial linear space).

In this section we first prove the following.

Theorem 3.1 Let $\Gamma=(\mathcal{P}, \mathcal{L})$ be a point-line geometry with point-collinearity graph $\Delta$. Suppose $\Gamma$ has thick lines. Let $(\mathcal{F}, \subseteq)$ be a poset of subsets of $\mathcal{P}$ ordered by inclusion. Suppose $\mathcal{F}$ satisfies the following conditions.

(F1) For every $S \in \mathcal{F}, S$ is a subspace of $\Gamma$ and $|S| \geq 2$.

(F2) $\mathcal{F}$ is closed under taking intersections, as long as the resulting intersection has cardinality at least two.

(F3) $\mathcal{L} \subseteq \mathcal{F}$.

Then the minimal elements of $\mathcal{F}$ containing at least three points are (1) the lines of $\Gamma$, and (2) the cocliques $C \in \mathcal{F}$ of $\Delta$, with $|C| \geq 3$ and such that every triple of distinct points of $C$ is an $\mathcal{F}$-round-up triple.

We break up the proof of this theorem into three lemmas and a conclusion.

Lemma 3.2 Let $\mathcal{P}$ be a set and let $(\mathcal{F}, \subseteq)$ be a poset of subsets of $\mathcal{P}$ ordered by inclusion. Suppose $\mathcal{F}$ satisfies (F2) of Theorem 3.1. If $X \in \mathcal{F}$ and $|X| \geq 3$, then $X$ is a minimal element of $(\mathcal{F}, \subseteq)$ if and only if every 3-element subset of $X$ is an $\mathcal{F}$-round-up triple.

Proof. Let $X \in \mathcal{F}$ be such that $|X| \geq 3$. 
Suppose first that $X$ is minimal in $\mathcal{F}$ and let $T$ be a 3-element subset of $X$. Suppose $Y \in \mathcal{F}$ is such that $|Y \cap T| \geq 2$. Then $|Y \cap X| \geq 2$, therefore by (F2) $Y \cap X \in \mathcal{F}$. By the minimality of $X$ this implies that $X \subseteq Y \cap X$. Therefore $T \subseteq Y$.

Suppose now that every 3 -element subset of $X$ is an $\mathcal{F}$-round-up triple and let $Y \in \mathcal{F}$ be such that $Y \subsetneq X$. Let $x \in X \backslash Y$ and let $\{y, z\}$ be a 2-element subset of $Y$, the latter exists by (F2). Then $\{x, y, z\}$ is a subset of $X$ of cardinality 3 which is not an $\mathcal{F}$-round-up triple, a contradiction. Therefore $X$ must be minimal.

Lemma 3.3 Let $\Gamma=(\mathcal{P}, \mathcal{L})$ be a point-line geometry. Let $(\mathcal{F}, \subseteq)$ be a poset of subsets of $\mathcal{P}$ ordered by inclusion. Suppose $\mathcal{F}$ satisfies $(\mathrm{F} 1)$ of Theorem 3.1. If $L \in \mathcal{L} \cap \mathcal{F}$, then every subset of $L$ of cardinality 3 is an $\mathcal{F}$-round-up triple.

Proof. Let $L \in \mathcal{L} \cap \mathcal{F}$ and let $T \subseteq L$ be such that $|T|=3$. Suppose $Y \in \mathcal{F}$ and $|Y \cap T|=2$. By (F1) $Y$ is a subspace of $\Gamma$, therefore $T \subseteq L \subseteq Y$.

Lemma 3.4 Suppose $\Gamma=(\mathcal{P}, \mathcal{L})$ is a point-line geometry with point-collinearity graph $\Delta$. Let $(\mathcal{F}, \subseteq)$ be a poset of subsets of $\mathcal{P}$ ordered by inclusion. Suppose $\mathcal{F}$ satisfies (F3) of Theorem 3.1. If $T$ is an $\mathcal{F}$-round-up triple then either $(i) T$ is a clique of $\Delta$ contained in a line of $\Gamma$ or, else, (ii) $T$ is a coclique of $\Delta$.

Proof. Suppose $\{p, q\} \subseteq T$ are distinct collinear points of $\Gamma$ and let $L=\langle p, q\rangle$. Then $|L \cap T| \geq 2$. By $($ F3) $L \in \mathcal{F}$, therefore $T \subseteq L$.

Proof of Theorem 3.1. By Lemmas 3.3 and 3.2, and since the lines of $\Gamma$ are thick, the subsets of $\mathcal{P}$ described in the conclusion are minimal elements of $\mathcal{F}$ of cardinality at least 3. It remains to show that every minimal element of $\mathcal{F}$ of cardinality at least 3 is either a line of $\Gamma$ or a coclique of $\Delta$, and this follows from Lemma 3.4 combined with Lemma 3.2.

We will apply Theorem 3.1 in Sections 5 and 6 to parapolar spaces arising from spherical buildings.

\subsection{Geometries arising from spherical buildings}

In this subsection we use the following notation.

Let $\mathcal{B}$ be a building of a spherical type $M$, a Coxeter diagram over a type set $I$. Suppose $J \subseteq I$ and $I^{\prime}=I-J$. The $J$-Grassmann geometry $\Gamma=(\mathcal{P}, \mathcal{L})$ is a point-line geometry whose points $p \in \mathcal{P}$ are the residues of $\mathcal{B}$ of type $I^{\prime}$. In this section, we consider residues as sets of chambers of $\mathcal{B}$, hence viewing buildings as chamber systems. The lines $L \in \mathcal{L}$ of $\Gamma$ are the point shadows of the panels of $\mathcal{B}$ of all possible cotypes $\{j\} \subseteq J$, i.e., the sets of residues of type $I^{\prime}$ meeting a panel nontrivially (viewing a panel as a set of chambers).

Given $L \in \mathcal{L}$, more than one panel of $\mathcal{B}$ can have the point shadow $L$. More precisely, suppose $P$ is a panel of $\mathcal{B}$ whose point shadow is $L$. Then $P$ is of type $\{j\} \subseteq J$. Let $T=\{j\} \cup\left[I^{\prime}-D_{0,1}(j)\right]$, where $D_{0,1}(j)$ denotes the set consisting of $j$ and all nodes $i \in I$ of the diagram $M$ connected to the node $j$ by at least one bond, and let $R$ be the residue 
of $\mathcal{B}$ of type $T$ containing $P$. The panels of $\mathcal{B}$ whose point shadow is $L$ are precisely the panels of $R$ of type $\{j\}$. The residue $R$ is the largest by inclusion residue of $\mathcal{B}$ with point shadow $L$ (see [12] Chapter 12; [9] Chapter 5; [8]). The residue $R$ can also be described as the largest by inclusion residue of $\mathcal{B}$ containing $P$ and having the form $P \times P^{\prime}$, where $\times$ denotes the direct product of chamber systems and $P^{\prime}$ is a residue of $\mathcal{B}$, intersecting $P$, whose type is contained in $I^{\prime}$. For a point or line $z \in \mathcal{P} \cup \mathcal{L}$, we denote $\mathrm{R}_{z}$ the residue of $\mathcal{B}$ corresponding to $z$. Also, the type of a residue $R$ will be denoted by $\operatorname{typ}(R)$.

In the sequel, we will refer to Hypothesis $(\mathbf{A})$ when $\Gamma=(\mathcal{P}, \mathcal{L})$ is the $J$-Grassmann geometry of the spherical building $\mathcal{B}$, and we denote $\Delta$ the point collinearity graph of $\Gamma$. Moreover, we assume that the sets $J$ and $I^{\prime}$ are stabilized by the opposition involution of $M$. If the latter is not satisfied, we call it Hypothesis $\left(\mathbf{A}^{*}\right)$ and we denote $I^{\prime \prime}$ the image of $I^{\prime}$ under the opposition involution.

We need the following two results of Blok and Brouwer that originally were stated for $\{j\}$-Grassmannians, but the same proofs go through for general $J$-Grassmannians.

Proposition 3.5 (Proposition 7.1 of [3]) Suppose Hypothesis (A*) holds and let $R$ be a residue of $\mathcal{B}$ of type $I^{\prime \prime}$. Then the set of residues of $\mathcal{B}$ of type $I^{\prime}$ not opposite to $R$ is a geometric hyperplane of $\Gamma$.

Suppose Hypothesis $\left(\mathrm{A}^{*}\right)$ holds. For a triple $\{P, Q, R\}$ of distinct residues of $\mathcal{B}$ of type $I^{\prime}$, we say that $\{P, Q, R\}$ is a round-up triple of $\mathcal{B}$ if no residue of $\mathcal{B}$ is opposite to exactly one of $P, Q, R$. For a residue $R$ of $\mathcal{B}$ of type $I^{\prime \prime}$, we denote $H_{\mathcal{B}, R}$, or just $H_{R}$, the set of all residues of $\mathcal{B}$ of type $I^{\prime}$ not opposite to $R$. By Proposition $3.5 H_{\mathcal{B}, R}$ is a hyperplane of $\Gamma$. We let $\mathcal{H}_{\mathcal{B}, I^{\prime}}=\left\{H_{\mathcal{B}, R} \mid R\right.$ residue of $\mathcal{B}$ of type $\left.I^{\prime \prime}\right\}$ and we let $\overline{\mathcal{H}}_{\mathcal{B}, I^{\prime}}$ be the set of all subsets of $\mathcal{P}$ containing at least two points and having the form $\cap X, X \subseteq \mathcal{H}_{\mathcal{B}, I^{\prime}}$.

Proposition 3.6 (Lemma 6.4 of [3]) Suppose Hypothesis (A*) holds and let $R$ be a residue of $\mathcal{B}$ with point shadow $S$.

(i) Suppose $Q$ is a residue of $\mathcal{B}$ of type $I^{\prime \prime}$ and suppose $S-H_{Q} \neq \emptyset$. Then $S \cap H_{Q}$ is a geometric hyperplane of $\Gamma \mid S$ consisting of the points $p \in S$, such that $\mathrm{R}_{p} \cap R$ is not opposite to $\operatorname{proj}_{R}(Q)$ in the building $Q$.

(ii) Suppose $Q^{\prime}$ is a residue of $R$ of type opposite to $I^{\prime} \cap \operatorname{typ}(R)$. Let $H^{\prime}$ be the set of points $p \in S$ such that $\mathrm{R}_{p} \cap R$ is not opposite to $Q^{\prime}$ in the building $R$. Then $H^{\prime}$ is a geometric hyperplane of $\Gamma \mid S$ and there exists a residue $Q$ of $\mathcal{B}$ of type $I^{\prime \prime}$ such that $\operatorname{proj}_{R}(Q)=Q^{\prime}$ and $H^{\prime}=S \cap H_{Q}$.

Lemma 3.7 Suppose Hypothesis $\left(\mathrm{A}^{*}\right)$ holds. Then the round-up triples of $\Gamma$ are precisely the $\overline{\mathcal{H}}_{\mathcal{B}, I^{\prime}}$-round-up triples of $\mathcal{P}$.

In Propositions 3.8-3.11 we look at the relationship of hyperplanes $H_{R}$, and round-up triples of $\Gamma$, with residues of $\mathcal{B}$.

Suppose $G=(V, E)$ is a graph, let $H=\left(V^{\prime}, E^{\prime}\right)$ be a subgraph of $G$, and let $x \in V$. We say that $H$ is strongly gated in $G$ with respect to $x$ if there exists $g \in V$ such that, for all $y \in V^{\prime}, \mathrm{d}_{G}(x, y)=\mathrm{d}_{G}(x, g)+\mathrm{d}_{H}(g, y)$. The vertex $g$ is called the gate of $x$ in $H$. The 
residues of a building are strongly gated with respect to the chambers of the building; the gate of a chamber $c$ in a residue $R$ is called the projection of $c$ on $R$ and is denoted $\operatorname{proj}_{R}(c)$.

Proposition 3.8 Suppose Hypothesis $\left(\mathrm{A}^{*}\right)$ holds. Let $S \subseteq \mathcal{P}$ be the point shadow of a residue $R$ of $\mathcal{B}$. Then, for every $p \in \mathcal{P}-S$, there exists $q \in \mathcal{P}$ such that $S \subseteq H_{q}$ and $p \in \mathcal{P}-H_{q}$.

Proof. Let $p \in \mathcal{P}-S$. Let $P=\mathrm{R}_{p}$ and let $x$ be a chamber in $P$. Let $g=\operatorname{proj}_{R}(x)$ and let $w_{1}=(x, \ldots, g)$ be a geodesic from $x$ to $g$ in $\mathcal{B}$. Let $h$ be a chamber of $R$ at a maximal distance from $g$ (that is, $g$ and $h$ are opposite in $R$ ) and let $w_{2}=(g, \ldots, h)$ be a geodesic from $g$ to $h$ in $R$.

The gallery $w_{1} \circ w_{2}$ is a geodesic from $x$ to $h$ in $\mathcal{B}$ and can be extended to a geodesic $w=w_{1} \circ w_{2} \circ w_{3}$ in $\mathcal{B}$, where $w_{3}$ is a geodesic beginning at $h$ and terminating at a chamber $y$ opposite to $x$ in $\mathcal{B}$. We have $h=\operatorname{proj}_{R}(y)$.

Let $Q$ be the residue of $\mathcal{B}$ of type $I^{\prime \prime}$ containing $y$. Since $x$ and $y$ are opposite in $\mathcal{B}$, we have $p \notin H_{Q}$. We claim that $S \subseteq H_{Q}$.

Let $A$ be the unique apartment of $\mathcal{B}$ containing $x$ and $y$. Since $A$ is convex in $\mathcal{B}$, all chambers of $w$ lie in $A$. Let $t \in S$ and let $T=\mathrm{R}_{t}$. Suppose, first, that $T \cap A \neq \emptyset$. Then, since $T$ is gated in $\mathcal{B}$ and $R$ and $A$ are convex in $\mathcal{B}$, we have $T \cap R \cap A \neq \emptyset$. Since $x$ is the unique chamber of $A$ opposite to $y$, the residue $P \cap A$ is the unique residue of $A$ opposite to $Q \cap A$. Since $p \notin S$, we have $T \neq P$, therefore $T \cap A$ is not opposite to $Q \cap A$. This shows that $t \in H_{Q}$.

Suppose now that $T \cap A=\emptyset$. Let $h^{\prime}=\operatorname{proj}_{(T \cap R)}(y)$ and let $v$ be a geodesic from $h$ to $h^{\prime}$ in $R$. Let $v_{A}$ be the unique gallery in $A$ that begins at $h$ and has the same type as $v$, let $h_{A}^{\prime}$ be the terminal chamber of $v_{A}$, and let $T_{A}$ be the residue of $\mathcal{B}$ of type $I-J$ containing $h_{A}^{\prime}$. Then $T_{A} \cap A \neq \emptyset$ and $T_{A} \cap R \neq \emptyset$, therefore by the first case $T_{A}$ and $Q$ are not opposite in $\mathcal{B}$. This implies that $T$ and $Q$ are not opposite in $\mathcal{B}$.

Corollary 3.9 Suppose the hypothesis of Proposition 3.8 holds. If $S \neq \mathcal{P}$, then $S=$ $\cap\left\{H_{Q} \mid Q \in X\right\}$ for some set $X$ of residues of $\mathcal{B}$ of type $I^{\prime \prime}$.

Corollary 3.10 Suppose the hypothesis of Proposition 3.8 holds. If $\{p, q, r\} \subseteq \mathcal{P}$ is a round-up triple of $\Gamma$ and $p, q \in S$, then $r \in S$.

Proposition 3.11 Suppose that Hypothesis (A*) holds. Let $R$ be a residue of $\mathcal{B}$ and let $S \subseteq \mathcal{P}$ be the point shadow of $R$. Suppose $\left\{p_{1}, p_{2}, p_{3}\right\} \subseteq S$ is a round-up triple of $\mathcal{B}$ and let $R_{i}=R \cap R_{p_{i}}$. Then $\left\{R_{1}, R_{2}, R_{3}\right\}$ is a round-up triple of the building $R$.

Proof. Let $I_{R}^{\prime}=I^{\prime} \cap \operatorname{typ}(R)$ and let $I_{R}^{\prime \prime}$ be the image of $I_{R}^{\prime}$ under the opposition involution of the diagram $M \mid \operatorname{typ}(R)$. Suppose by way of contradiction that $Q$ is a residue of $R$ not opposite to $R_{1}$ and $R_{2}$ and opposite to $R_{3}$.

Since $Q$ is opposite to $R_{3}$, we have $\operatorname{typ}(Q)=I_{R}^{\prime \prime}$. By Proposition 3.6 there is a residue $Q^{\prime}$ of $\mathcal{B}$ of type $I^{\prime \prime}$ such that $H_{R, Q}=\left\{Z \cap R \mid Z \in H_{\mathcal{B}, Q^{\prime}}\right.$ and $\left.Z \cap R \neq \emptyset\right\}$. Since $\left\{R_{1}, R_{2}\right\} \subseteq H_{R, Q}$ 
and $R_{3} \notin H_{R, Q}$, we obtain $\left\{\mathrm{R}_{p_{1}}, \mathrm{R}_{p_{2}}\right\} \subseteq H_{\mathcal{B}, Q^{\prime}}$ and $\mathrm{R}_{p_{3}} \notin H_{\mathcal{B}, Q^{\prime}}$, a contradiction, since $\left\{p_{1}, p_{2}, p_{3}\right\}$ is a round-up triple.

Let $\mathcal{B}$ be a building over a type set $I$ with Coxeter group $(W, I)$, and suppose $Q$ and $R$ are residues of $\mathcal{B}$. Let $\mathcal{W}_{Q R}$ be the set of all galleries in $\mathcal{B}$ connecting chambers of $Q$ to chambers of $R$, and let $w \in \mathcal{W}_{Q R}$ be a gallery of the smallest length among all galleries in $\mathcal{W}_{Q R}$. The type of $w$ is a word $t=\operatorname{typ}(w)$ in the free monoid of words on $I$. Let $\delta(Q, R)$ be the element of the group $W$ corresponding to the word $t$. Then $\delta(Q, R)$ depends only on $Q$ and $R$ and does not depend on the choice of the particular shortest gallery $w \in \mathcal{W}_{Q R}$ (see $[12])$.

Our next aim is to prove the following rather fundamental property of round-up triples.

Proposition 3.12 Suppose that Hypothesis (A*) holds. Let $T=\left\{p_{1}, p_{2}, p_{3}\right\}$ be a roundup triple and let $R_{i}=\mathrm{R}_{p_{i}}$. Then $\delta\left(R_{i}, R_{j}\right)=\delta\left(R_{1}, R_{2}\right)$ for all pairs $\{i, j\} \subseteq\{1,2,3\}$.

Let $\mathcal{B}$ be a building over a type set $I$. Suppose $A$ is an apartment of $\mathcal{B}$ and suppose $x$ is a chamber of $A$. Then $\operatorname{retr}_{A, x}: \mathcal{B} \rightarrow A$ is the retraction of $\mathcal{B}$ onto $A$ with center $x$ defined in [12]; see also [10]. The retraction $\operatorname{retr}_{A, x}$ is a distance contracting map. For a gallery $w$ in $\mathcal{B}, \operatorname{typ}(w)$ denotes the type of $w ; \operatorname{typ}(w)$ is a word in the free monoid of words on $I$ consisting of the types of the arcs of $w$.

Our proof of Proposition 3.12 relies on the following lemma.

Lemma 3.13 Suppose that Hypothesis $\left(\mathrm{A}^{*}\right)$ holds. Let $T=\left\{p_{1}, p_{2}, p_{3}\right\}$ be a round-up triple and let $R_{i}=R_{p_{i}}$. Suppose $A$ is an apartment of $\mathcal{B}$ such that $A \cap R_{1} \neq \emptyset$ and $A \cap R_{2} \neq \emptyset$, and let $x$ be a chamber of $A$ opposite to a chamber of $A \cap R_{1}$. Then $\operatorname{retr}_{A, x}\left(R_{3}\right)=A \cap R_{1}$.

Proof. Let $Q_{1}=R_{1} \cap A$ and let $Q_{1}^{\prime}$ be the unique residue of $A$ opposite to $Q_{1}$. Since $A$ is thin and $Q_{1}=A \cap R_{1} \neq A \cap R_{2}$, the residues $Q_{1}^{\prime}$ and $A \cap R_{2}$ of $A$ are not opposite to each other in $A$.

Let $R_{1}^{\prime}$ be the residue of $\mathcal{B}$ of type $I^{\prime \prime}$ containing $Q_{1}^{\prime}$. Then $R_{1}^{\prime}$ is opposite to $R_{1}$ and is not opposite to $R_{2}$ in $\mathcal{B}$. Since $T$ is a round-up triple, $R_{1}^{\prime}$ must be opposite to $R_{3}$ in $\mathcal{B}$.

We have $x \in Q_{1}^{\prime} \subseteq R_{1}^{\prime}$. Therefore $R_{3}$ contains a chamber $x^{\prime}$ opposite to $x$ in $\mathcal{B}$. This implies that $\operatorname{retr}_{A, x}\left(R_{3}\right)$ contains a chamber $x^{\prime \prime}$ opposite to $x$ in $A$ and, therefore, $\operatorname{retr}_{A, x}\left(R_{3}\right)$ is opposite to $Q_{1}^{\prime}$ in $A$. Since $A$ is thin, $\operatorname{retr}_{A, x}\left(R_{3}\right)=Q_{1}$.

For an element $w$ of a Coxeter group $(W, I)$, we denote $l(w)$ the length of $w$, that is the number of terms in the shortest word over $I$ representing $w$.

Proof of Proposition 3.12. It suffices to show that $\delta\left(R_{1}, R_{2}\right)=\delta\left(R_{2}, R_{3}\right)$ and the result will follow by symmetry.

Let $A$ and $x$ be as in Lemma 3.13. Then $\operatorname{retr}_{A, x}\left(R_{2}\right)=A \cap R_{2}$ and by Lemma 3.13 $\operatorname{retr}_{A, x}\left(R_{3}\right)=A \cap R_{1}$. Let $w$ be a shortest gallery among the galleries in $\mathcal{W}_{R_{2} R_{3}}$, and let $w^{\prime}=\operatorname{retr}_{A, x}(w)$ be the nonstammering gallery which is the image of $w$ under $\operatorname{retr}_{A, x}$. Then $w^{\prime} \in \mathcal{W}_{R_{2} R_{1}}$. 
Consider the hypothesis of Lemma 3.13 with the roles of $R_{1}$ and $R_{3}$ interchanged and with $A$ and $x$ renamed $A^{\prime}$ and $x^{\prime}$. Let $w^{\prime \prime}=\operatorname{retr}_{A^{\prime}, x^{\prime}}\left(w^{\prime}\right)$ be the image of $w^{\prime}$ under $\operatorname{retr}_{A^{\prime}, x^{\prime}}$. By Lemma $3.13 w^{\prime \prime} \in \mathcal{W}_{R_{2} R_{3}}$. By a property of retractions (see [12] or [10]), $\operatorname{typ}\left(w^{\prime}\right)$ is obtained from $\operatorname{typ}(w)$, and $\operatorname{typ}\left(w^{\prime \prime}\right)$ is obtained from $\operatorname{typ}\left(w^{\prime}\right)$, by omiting a number of terms, possibly zero. Since $w$ is a shortest gallery in $\mathcal{W}_{R_{2} R_{3}}$, we obtain $l\left(w^{\prime \prime}\right)=1(w)$. Therefore $\operatorname{typ}\left(w^{\prime \prime}\right)=\operatorname{typ}\left(w^{\prime}\right)=\operatorname{typ}(w)$.

We will use Propositions 3.12 in the following form.

Corollary 3.14 Suppose that Hypothesis ( $\left.\mathrm{A}^{*}\right)$ holds. Let $T=\left\{p_{1}, p_{2}, p_{3}\right\}$ be a round-up triple. Then $\mathrm{d}_{\Delta}\left(p_{i}, p_{j}\right)=\mathrm{d}_{\Delta}\left(p_{1}, p_{2}\right)$ for all pairs $\left\{p_{i}, p_{j}\right\} \subseteq\left\{p_{1}, p_{2}, p_{3}\right\}$. Furthermore, if $\Gamma$ is a parapolar space and $\mathrm{d}_{\Delta}\left(p_{1}, p_{2}\right)=2$, then either all pairs $\left\{p_{i}, p_{j}\right\} \subseteq\left\{p_{1}, p_{2}, p_{3}\right\}$ are symplectic or, else, they all are special.

Suppose Hypothesis $\left(\mathrm{A}^{*}\right)$ holds. We say that a subset $L \subseteq \mathcal{P}$ is a geometric line of $\mathcal{B}$ (or of $\Gamma$ ) if any residue of $\mathcal{B}$ of type $I^{\prime \prime}$ is non-opposite either to all of $\mathrm{R}_{p}$, with $p \in L$, or to exactly one of $\mathrm{R}_{p}$, with $p \in L$.

Let $L$ be a geometric line. It is straightforward to see that every triple in $L$ is a round-up triple. Hence we can state:

Corollary 3.15 Suppose that Hypothesis $\left(\mathrm{A}^{*}\right)$ holds. Let $L$ be a geometric line, and let $p, q \in L$. Then $\mathrm{d}_{\Delta}(x, y)=\mathrm{d}_{\Delta}(p, q)$ for all pairs $\{x, y\} \subseteq L$. Furthermore, if $\Gamma$ is a parapolar space, $\{p, q\}$ is a symplectic pair, and $L$ is contained in a symplecton, then $L$ is a geometric line in that symplecton (viewed as a building).

\section{Polar spaces}

\subsection{Statement and Strategy}

The aim of this section is to show the following theorem.

Theorem 4.1 Let there be given all pairs of opposite vertices of a certain (unknown) self-opposite type $t$ of the (not necessarily thick) building $\mathcal{B}$ associated to an (unknown) polar space $\Omega$ with thick lines of finite (unknown) rank, or to its oriflamme complex. Then there is a mathematical algorithm that allows to determine the isomorphism class of $\Omega$ and the type $t$, up to a diagram automorphism, except that, if $t$ is the type of the maximal singular subspaces of a polar space associated to a non-degenerate quadratic form of maximal Witt index $2 n-1$ in a $(4 n-2)$-dimensional projective space, $n \geq 2$, then the algorithm tells us that $t$ is the type of one of the families of maximal singular subspaces of a polar space associated to a non-degenerate quadratic form of maximal Witt index $2 n$ in $a(4 n-1)$-dimensional projective space, and the associated building $\Omega$ is necessarily the corresponding oriflamme complex.

Hence, if $t$ is not the type of the maximal singular subspaces of a polar space associated to a non-degenerate quadratic form of maximal Witt index $2 n-1$ in a $(4 n-2)$-dimensional 
projective space, $n \geq 2$, then every permutation of the set of vertices of type $t$ of $\mathcal{B}$ preserving opposition and non-opposition is induced by a collineation of $\Omega$, except if $\Omega$ is a polar space of type $\mathrm{D}_{4}$ and (1) the vertices under consideration are the lines of $\Omega$, or (2) the vertices under consideration are of end-node type, but not the points.

In the former case (1), which we can detect from the given pairs of opposite vertices, every permutation of the set of lines preserving opposition and non-opposition is induced by an automorphism of the building of type $\mathrm{D}_{4}$ corresponding to $\Omega$ (and that automorphism might be non-type preserving, in particular, trialities are allowed).

In the latter case (2), which we can also detect from the given pairs, every permutation of the given set of vertices preserving opposition and non-opposition is induced by an automorphism of the building of type $\mathrm{D}_{4}$ corresponding with $\Omega$, but this time the automorphism, preserving type $t$, can only be type preserving, or a duality.

The maximal singular subspaces of polar spaces associated to non-degenerate quadratic forms of maximal Witt index in $(4 n-2)$-dimensional projective spaces are a true exception to the theorem. Indeed, consider such a polar space $\Omega$ of rank $2 n-1$. Then $\Omega$ corresponds to a quadric of Witt index $2 n-1$ in some projective space $\mathrm{PG}(4 n-2, \mathbb{K})$, with $\mathbb{K}$ some field. Embed $\Omega$ into a hyperbolic quadric $\Omega^{\prime}$ of rank $2 n$, living in $\mathrm{PG}(4 n-1, \mathbb{K})$. Let $\mathcal{G}$ be one of the classes of maximal singular subspaces of $\Omega^{\prime}$. There is a bijection $\beta$ attaching to each maximal subspace $S$ of $\Omega$ the unique member of $\mathcal{G}$ containing $S$. Moreover, since the rank of $\Omega^{\prime}$ is even, we have that, for each pair $\left(S, S^{\prime}\right)$ of maximal singular subspaces of $\Omega, S$ is opposite $S^{\prime}$ if and only if $\beta(S)$ is opposite $\beta\left(S^{\prime}\right)$. Hence every automorphism $\alpha$ of $\Omega^{\prime}$ preserving $\mathcal{G}$ induces a permutation $S \mapsto \beta^{-1}(\alpha(\beta(S)))$ on the set of maximal singular subspaces of $\Omega$ preserving opposition. If $\alpha$ does not stabilize $\Omega$, then clearly this permutation cannot be induced by an automorphism of $\Omega$.

Let us also remark that we cannot always reconstruct $\mathcal{B}$ since, if $\Omega$ is non-thick, there are essentially two choices for $\mathcal{B}$ : a non-thick building, or the oriflamme complex. The reason we want to include the non-thick case here is for the next-to-maximal singular subspaces, which are not elements of the building in the oriflamme case.

The strategy of the proof is as follows. We first classify the round-up triples of vertices of type $t$ in all buildings arising from polar spaces or their oriflamme complexes. Such triples will usually be collinear points in the $t$-Grassmannian, but in some cases, also other triples turn up. Then we determine precisely in which cases round-up triples give rise to geometric lines that are not lines in the $t$-Grassmannian. In order to distinguish between these, we determine maximal cliques in the "geometric collinearity graph", defined by these geometric lines. Looking at the behaviour of these cliques with respect to these triples, we will be able to recover the $t$-Grassmannian. Together with opposition, this will enable us to identify the vertices of type $t-1$ (or, in some cases, those of type $t-2$ ), and determine the corresponding opposition relation. Continuing this procedure, we will eventually get the point set of $\Omega$, exactly when non-opposition defines the collinearity relation of a polar space, which must necessarily be $\Omega$. In the course of the proof, we will recognize a case met exactly by the polar spaces of type $D_{4}$. We will have to treat this case separately. 


\subsection{Preliminary lemmas}

We now embark on the proof of Theorem 4.1. Throughout, let $\Omega$ be a polar space of rank $r$ at least 3, with thick lines and let $i$ be such that $0 \leq i<r$. For the time being, we do not put any restriction on $\Omega$. We use common terminology and notation, such as $A^{\perp}$ for the set of points of $\Omega$ collinear to all points of the set $A$ (which is also supposed to be a set of points). We use one, perhaps less standard, convention, and that is, for a non-maximal singular subspace $U$, we denote by $\operatorname{Res}_{\Omega}(U)$ the polar space obtained by considering all singular subspaces of $\Omega$ which properly contain $U$.

We denote by $\mathcal{B}$ a building corresponding to $\Omega$. The building $\mathcal{B}$ is unique if $\Omega$ is thick, but there are two choices if $\Omega$ is non-thick: the canonical non-thick building, or the oriflamme complex, see [12].

Let $U, V, W$ be three singular subspaces of dimension $i$ of $\Omega$. Recall that $\{U, V, W\}$ is a round-up triple if the subspaces $U, V, W$ have the same dimension, and if no singular subspace is opposite exactly one of $U, V, W$.

We want to determine all round-up triples of singular subspaces of dimension $i$ of $\Omega$. We start with a lemma stating an equivalent, workable condition for being a round-up triple in a polar space.

Lemma 4.2 Let $U, V, W$ be three singular subspaces of dimension $i$ of the polar space $\Omega$. Then $\{U, V, W\}$ is a round-up triple if and only if the following two conditions are satisfied.

(RU1) We have $U^{\perp} \cap V^{\perp}=V^{\perp} \cap W^{\perp}=W^{\perp} \cap U^{\perp}$.

(RU2) Whenever a line $L$ contains two distinct points $u, v$ with $U \subseteq u^{\perp}$ and $V \subseteq v^{\perp}$, then there is a point $w \in L$ with $W \subseteq w^{\perp}$.

Proof. First suppose that $\{U, V, W\}$ is a round-up triple. To prove (RU1), we may suppose without loss of generality, and by way of contradiction, that there is a point $x \in\left(U^{\perp} \cap V^{\perp}\right)-W^{\perp}$. Then $x \notin W$ and we can include $x$ in a singular subspace $W^{\prime}$ opposite $W$. Since $x$ is collinear with all points of both $U$ and $V$, the subspace $W^{\prime}$ can neither be opposite $U$ nor $V$.

To prove (RU2), we may assume, by way of contradiction, that a line $L$ contains two distinct points $u, v$ with $U \subseteq u^{\perp}$ and $V \subseteq v^{\perp}$, and with $W^{\perp} \cap L=\emptyset$. The latter implies that there is a singular subspace $W^{\prime}$ containing $L$ and opposite $W$. As in the previous paragraph, this leads to the contradiction that $W^{\prime}$ is not opposite $U$ nor $V$.

Now suppose $U, V, W$ are three singular subspaces satisfying both (RU1) and (RU2). By assumption, these have the same dimension.

Let $W^{\prime}$ be a singular subspace opposite $W$ but not opposite $U$ nor $V$. Since $U, V, W^{\prime}$ have the same type, there are points $u, v \in W^{\prime}$ collinear with all points of $U$ and $V$, respectively. By (RU1), the points $u$ and $v$ are distinct. But they are clearly collinear, 
and so there is a point $w$ on the line $\langle u, v\rangle$, and hence in $W^{\prime}$ collinear with all points of $W$, contradicting the fact that $W^{\prime}$ is opposite $W$.

So, from now on, when we have a round-up triple $\{U, V, W\}$, we will freely use the Conditions (RU1) and (RU2) of Lemma 4.2.

The surprising, nice and very useful thing about (RU1) and (RU2) is that these are residual properties, whereas the definition of round-up is far from residual, since no two singular subspaces in a proper residue can be opposite each other! This is, however, in accordance with Proposition 3.11. We make this more concrete with the following lemma which completes Proposition 3.11 to an "if-and-ony-if"-statement in this particular case.

Lemma 4.3 Let $U, V, W$ be three singular subspaces. Let $p$ be a point of $\Omega$ in $U \cap V \cap W$. Then $\{U, V, W\}$ satisfies Conditions (RU1) and (RU2) if and only if $U, V, W$, viewed as subspaces of the residue of $\Omega$ in p satisfy Conditions (RU1) and (RU2) inside $\operatorname{Res}_{\Omega}(p)$.

Proof. The assertion follows easily from the fact that collinearity of points in $\operatorname{Res}_{\Omega}(p)$ is equivalent with the corresponding lines of $\Omega$ being coplanar, noting that, for (RU2), if two points of a line of $\Omega$ are contained in $U^{\perp}$ and $V^{\perp}$, respectively, then every point of that line is collinear with $p$.

The next lemma deals with the case where a round-up triple is contained in a common singular subspace.

Lemma 4.4 Let $\{U, V, W\}$ be a round-up triple such that $U$ and $V$ are contained in a (minimal) common singular subspace $Z$. Then also $W$ is contained in $Z$, and we have $1+\operatorname{dim} U=\operatorname{dim} Z$ and $U \cap V=V \cap W=W \cap U$.

Proof. Considering the residue of $Z$ in the building $\mathcal{B}$, Corollary 3.10 implies that $W$ is contained in $Z$. Hence, by Proposition 3.11, $\{U, V, W\}$ is a round-up triple in the projective space $Z$. The assertion now follows from Subsection 5.2.

Lemma 4.5 Let $\{U, V, W\}$ be a round-up triple. Then $U \cap V=V \cap W=W \cap U$.

Proof. By symmetry, it suffices to prove that $U \cap V \subseteq W$. Considering the residue of $U \cap V$ in $\mathcal{B}$, this follows from Corollary 3.10.

Lemma 4.6 Let $U, V, W$ be maximal singular subspaces satisfying Conditions (RU1) and (RU2). Then $2+\operatorname{dim}(U \cap V) \geq \operatorname{dim} U$.

Proof. Suppose, by way of contradiction, that $2+\operatorname{dim}(U \cap V)<\operatorname{dim} U$, with $U, V, W$ maximal subspaces satisfying (RU1) and (RU2). In the residue $\operatorname{Res}_{\Omega}(U \cap V)$, the subspaces $U, V$ and $W$ are opposite maximal singular subspaces (using Lemma 4.5). Hence we may assume, using Lemma 4.3, that $U, V, W$ are mutually opposite maximal singular subspaces, and that the rank of $\Omega$ is at least 3 .

Let $u$ be any point of $U$. Since the rank of $\Omega$ is at least 3, there is a plane $\pi$ through $u$ meeting $V$ in a line $L$. If $\pi$ met $W$ in a line $M$, then $L \cap M$ would belong to $V \cap W$, which 
is empty, hence we obtain a contradiction. Consequently $|\pi \cap W| \leq 1$. This implies that we can choose a line $K$ through $u$ not meeting $W$. Since $K$ meets $L$, say in the point $v$, we have a line $\langle u, v\rangle$ with a point $u \in U^{\perp}=U$ and a point $v \in V^{\perp}=V$, but containing no point in (the perp of) $W$. Hence $U, V, W$ cannot satisfy (RU2), a contradiction.

Lemma 4.7 Let $U, V, W$ be nonmaximal singular subspaces, satisfying Conditions (RU1) and (RU2). Then $1+\operatorname{dim}(U \cap V) \geq \operatorname{dim} U$.

Proof. Suppose, by way of contradiction, that $1+\operatorname{dim}(U \cap V)<\operatorname{dim} U$, with $U, V, W$ nonmaximal subspaces satisfying (RU1) and (RU2). In the residue $\operatorname{Res}_{\Omega}(U \cap V)$, the subspaces $U, V$ and $W$ are pairwise disjoint nonmaximal singular subspaces (using Lemma 4.5) of dimension at least 1 . Hence we may assume, using Lemma 4.3, that $U, V, W$ are pairwise disjoint nonmaximal singular subspaces of dimension at least 1 , and that the rank of $\Omega$ is at least 3. Moreover, by Lemma 4.4, we may assume that $U, V, W$ are not contained in a common subspace (which by Condition (RU1) again implies that they are pairwise not contained in a singular subspace).

Let $V_{U}$ and $W_{U}$ be the set of points of $V$ and $W$, respectively, collinear with all points of $U$, and set $U^{V}=\left\langle U, V_{U}\right\rangle$ and $U^{W}=\left\langle U, W_{U}\right\rangle$. In $\operatorname{Res}_{\Omega}(U)$ we can find a point not collinear with all points of the respective singular subspaces $U^{V}$ and $U^{W}$ (using the fact that $\operatorname{Res}_{\Omega}(U)$ is a polar space with thick lines). Let $U^{\prime}$ be a singular subspace of dimension $1+\operatorname{dim} U$, containing $U$, corresponding with such point of $\operatorname{Res}_{\Omega}(U)$, and let $u^{\prime}$ be a point of $\Omega$ contained in $U^{\prime}-U$. Interchanging the roles of $U$ and $V$, we construct a singular subspace $V^{\prime}$ with similar properties, but with $U$ and $V$ interchanged. It follows from these properties that the subspace $V^{\prime} \cap W^{\perp}$ has dimension at most $-1+\operatorname{dim} V$.

Now let $Z$ be a singular subspace of dimension $\operatorname{dim} V^{\prime}$ containing $u^{\prime}$ and intersecting $V^{\prime}$ in a hyperplane $H$ of $V^{\prime}$. The hyperplane $H$ cannot coincide with $V$ as $u^{\prime}$ is not collinear with all points of $V_{U} \subseteq V$ by the choice of $U^{\prime}$. By the same token, $V_{U} \nsubseteq H$. Now, from Condition (RU1) follows that $V_{U}=V_{W}$; also, $W^{\perp}$ does not contain $V \cap H$, as the latter is a hyperplane in $V$ and otherwise $W^{\perp}$ would contain $V \cap H$ and $V_{U}$, which span the whole of $V$, a contradiction. All this implies that $H \cap W^{\perp}$ has dimension at most $-2+\operatorname{dim} H$. This implies that $W^{\perp} \cap Z$ has dimension at most $-2+\operatorname{dim} Z$. Hence we can find a line $L$ in $Z$ containing $u^{\prime}$ disjoint from $W^{\perp}$. But $u^{\prime} \in U^{\perp}$ and the intersection point $L \cap H$ belongs to $V^{\perp}$ ( $L$ indeed intersects $H$ nontrivially as $H$ is a hyperpane of $Z$ ). This contradicts Condition (RU2) and the assertion follows.

Hence, denoting the intersection of $U, V, W$ by $C$, we have the following cases for a roundup triple $\{U, V, W\}$.

(NM1) $U, V, W$ are collinear points in $\operatorname{Res}_{\Omega}(C)$, and $\operatorname{Res}_{\Omega}(C)$ has rank $\geq 2$.

(MS1) $U, V, W$ are distinct points in $\operatorname{Res}_{\Omega}(C)$, and $\operatorname{Res}_{\Omega}(C)$ has rank 1 .

(NM2) $U, V, W$ are opposite points in $\operatorname{Res}_{\Omega}(C), W \in\{U, V\}^{\perp \perp}$, and $\operatorname{Res}_{\Omega}(C)$ has rank $\geq 2$.

(MS2) $U, V, W$ are opposite lines in $\operatorname{Res}_{\Omega}(C), W$ meets every line that meets both $U$ and $V$, and $\operatorname{Res}_{\Omega}(C)$ has rank 2 . 
The geometric lines corresponding to (NM1) and (MS1) are just the lines of the corresponding Grassmannian, which we shall call Grassmannian lines. Now we determine precisely when the cases (NM2) and (MS2) give rise to geometric lines.

Lemma 4.8 A set $L$ of opposite points of a polar space $\Omega$ of rank $r \geq 2$ is a geometric line if and only if $\Omega$ corresponds to a symplectic polarity in some $\mathrm{PG}(2 n-1, \mathbb{K})$, for some field $\mathbb{K}$, and $L$ corresponds to an ordinary but non-singular line of $\mathrm{PG}(2 n-1, \mathbb{K})$.

Proof. Since two opposite points always lie in a rank 2 residue, it suffices to prove the lemma for $r=2$. So let $T$ be a set of opposite points constituting a geometric line. If a point $x$ is collinear with two elements of $T$, then all elements of $T$ are collinear with $x$, hence we see that $T$ is contained in a trace $\{x, y\}^{\perp}$. Suppose, by way of contradiction, that some line $L$ through $x$ does not contain any member of $T$. Then no point of $T$ is collinear with the point on $L$ collinear with $y$, contradicting the fact that $T$ is a geometric line. Hence $T=\{x, y\}^{\perp}$. From the definition of geometric line and the fact that the automorphism group of $\Omega$ is distance transitive now immediately follows that $x$, and hence every point, is regular. Since every point is non-opposite some point of $T$, or in other words $z^{\perp} \cap x^{\perp} \cap y^{\perp}$ is nonempty for every point $z$, the set $T$ is a long hyperbolic line, in the terminology of [13], see also [14]. Now [13], see also Theorem 6.5.3 of [14], concludes the proof of the lemma.

It now follows immediately that for $i<r-1$, the polar space $\Omega$ admits a geometric line which is not a Grassmannian line if and only if $\Omega$ arises from a symplectic polarity (then $\mathcal{B}$ is a split building of absolute and relative type $C_{n}$ ). For ease of reference, we will call such polar space a symplectic polar space.

Lemma 4.9 A set $L$ of opposite lines of a generalized quadrangle $\Omega$ with thick lines is a geometric line if and only if $\Omega$ is either a grid and $L$ consists of one class of generators, or $\Omega$ is the dual of a symplectic quadrangle and $L$ is as in the dualized rank 2 case of Lemma 4.8 .

Proof. If $\Omega$ is non-thick, then we clearly have a grid and the result follows trivially. If $\Omega$ is thick, then this follows from the dual of the previous lemma.

It now follows immediately that for $i=r-1$, the polar space $\Omega$, which we now assume to be thick, admits a geometric line which is not a Grassmannian line if and only if $\Omega$ is a quadric of maximal Witt index in even-dimensional projective space (then $\mathcal{B}$ is a split building of absolute and relative type $\mathrm{B}_{n}$ ). For ease of reference, we will call such a polar space a parabolic polar space.

We now define the following graph $\Delta_{i}=\left(Y_{i}, \sim\right)$, where $i<r$. The set $Y_{i}$ is the set of singular subspaces of $\Omega$ of dimension $i$, two vertices $U, V$ being adjacent if they are contained in a common geometric line. Our first aim is to determine all maximal cliques of this graph. In fact, we will treat this problem slightly more general, by noting that, if there exist round-up triples not contained in a Grassmannian line, then two distinct singular subspaces $U, V$ of dimension $i$ are adjacent if and only if (1) for $i<r-1$, $\operatorname{dim}(U \cap V)=-1+\operatorname{dim} U$, or (2) for $i=r-1, \operatorname{dim}(U \cap V) \geq-2+\operatorname{dim} U$. But first we treat the case where every round-up triple would be contained in a Grassmannian line. 
So let $\Delta_{i}^{\prime}$ be the graph on $Y_{i}$ where two distinct vertices $U, V$ are adjacent if $\operatorname{dim}(U \cap V)=$ $-1+\operatorname{dim} U$ and, in case $i<r-1$, then we also require that $U$ and $V$ are contained in a common maximal subspace.

Lemma 4.10 Any maximal clique of $\Delta_{i}^{\prime}, i<r-1$, either corresponds to the set of all singular subspaces of dimension $i$ contained in a fixed singular subspace of dimension $i+1$, or $i<r-2$ and then it can also correspond to the set of all singular subspaces of dimension $i$ contained in a maximal singular subspace and containing a fixed singular subspace of dimension $i-1$. If $i=r-1$, then any maximal clique corresponds to the set of maximal subspaces containing a fixed next-to-maximal singular subspace.

Proof. Suppose $i<r-1$. Let $U \sim V$ in $\Delta_{i}^{\prime}$. Then, by definition, all points of $U$ are collinear with all points of $V$. Hence, the union of all points of the subspaces of dimension $i$ corresponding to the vertices of $\Delta_{i}^{\prime}$ of a maximal clique $\mathcal{C}$, is a set of mutually collinear points in $\Omega$. Hence it is contained in a singular subspace $Z$, which we may assume to be of minimal dimension. If $\operatorname{dim} Z=i+1$, then clearly $\mathcal{C}$ consists of all subspaces of $Z$ of dimension $i$, and this is clearly a maximal clique. Suppose now $\operatorname{dim} Z>i+1$. Then there are three elements $U, V, W \in \mathcal{C}$ generating a subspace $S$ of dimension $i+2$. Clearly, $U \cap V=V \cap W=W \cap U$, and any other vertex $A \in \mathcal{C}$ must contain $U \cap V \cap W$. By maximality of the clique $\mathcal{C}$, it now follows easily that $Z$ is a maximal singular subspace (as every singular subspace is contained in a maximal one) and every member of $\mathcal{C}$ contains $U \cap V$.

Now suppose $i=r-1$. Let $\{U, V, W\}$ be a triple of pairwise adjacent vertices in $\Delta_{i}^{\prime}$. By definition, $U \cap V$ is $(i-1)$-dimensional. If $W$ does not contain $U \cap V$, then $(W \cap U)-(U \cap V)$ is nonempty, and any point $x$ in that set is collinear with all points of $(U \cap V) \cup((W \cap$ $V)-U)$. Since also $((W \cap V)-U)$ is nonempty, this implies that $x$ is collinear with all points of $V$, contradicting the assumption $x \notin V$. Hence $U \cap V=V \cap W=W \cap U$ and the assertion follows.

Now let $\Delta_{i}^{\prime \prime}$ be the graph on $Y_{i}$ where two distinct vertices $U, V$ are adjacent if $\operatorname{dim}(U \cap V)=$ $-1+\operatorname{dim} U$ when $i<r-1$, or $\operatorname{dim}(U \cap V) \geq i-2$ when $i=r-1$. We first treat the case of maximal singular subspaces.

Lemma 4.11 Any maximal clique in $\Delta_{i}^{\prime \prime}$, with $i=r-1$, corresponds either to the set of maximal singular subspaces containing a fixed singular subspace of dimension $i-2$, or to the set of all maximal singular subspaces intersecting a fixed maximal singular subspace in a singular subspace of dimension at least $i-1$, or every pair of members of the maximal clique intersects in a subspace of dimension $i-2$ and they all contain a fixed subspace of dimension $i-3$.

Proof. Let $Z$ be a fixed member of a given maximal clique $\mathcal{C}$ in $\Delta_{i}^{\prime \prime}$ and suppose that $Z$ is chosen in such a way that, if $\mathcal{C}$ contains maximal singular subspaces intersecting one another in a subspace of dimension $i-1$, then $Z$ is one of those.

Suppose first that there exist three members $U, V, W, \in \mathcal{C}$ intersecting $Z$ in distinct subspaces of dimension $i-1$ with $\operatorname{dim}(Z \cap U \cap V \cap W)=i-3$. Suppose there exists an 
$A \in \mathcal{C}$ intersecting $Z$ in a subspace $A^{\prime}$ of dimension $i-2$. Then our assumptions imply that $A^{\prime}$ is non-contained in at least one of $U, V, W$, say $U$. Hence there is a point $x$ of $U \cap A$ not in $Z$. Then $x$ is collinear with all points of $(Z \cap U) \cup(Z \cap A)$, which spans $Z$. This contradiction shows that $\mathcal{C}$ consists of all maximal singular subspaces of $\Omega$ which intersect $Z$ in a subspace of dimension at least $i-1$.

Next we assume that all members of $\mathcal{C}$ which intersect $Z$ in a hyperplane of $Z$ contain a fixed subspace $S$ of dimension $i-2$, and there are at least two members $U, V$ intersecting $Z$ in distinct hyperplanes. By assumption, all other members of $\mathcal{C}$ intersecting $Z$ in a hyperplane of $Z$, contain $S$, and by the same argument as in the previous paragraph, the intersection with $Z$ of every member of $\mathcal{C}$ intersecting $Z$ in a subspace of dimension $i-2$ must be contained in both $Z \cap U$ and $Z \cap V$, and so coincides with $S$. Consequently, every member of $\mathcal{C}$ contains $S$ and $\mathcal{C}$ consists of the set of all maximal singular subspaces containing $S$.

Next, we assume that there is a member $U$ of $\mathcal{C}$ intersecting $Z$ in a hyperplane $H$ of $Z$, and every other member of $\mathcal{C}$ intersecting $Z$ in a hyperplane of $Z$ contains $H$. Since, by the same token as before, every other member of $\mathcal{C}$ intersects $Z$ in a subspace contained in $H$, we deduce by the maximality of $\mathcal{C}$ that every maximal singular subspace of $\Omega$ containing $H$ belongs to $\mathcal{C}$. Since, again by the maximality of $\mathcal{C}$, the latter does not consist of all maximal singular subspaces of $\Omega$ through a singular subspace of dimension $i-2$ (as otherwise this subspace belongs to $H$ and we would be in the previous case), there exist two members $V, W \in \mathcal{C}$ with $Z \cap V \neq Z \cap W$ and with both intersections of dimension $i-2$ and contained in $H$. This implies that $Z \cap V \cap W$ has dimension $i-3$ and so there is some point $y \in V \cap W$ that does not belong to $Z$. But that point is collinear to all points of $Z \cap V$ and $Z \cap W$, and these points generate $H$. Hence $y$ belongs to a maximal singular subspace $A$ intersecting $Z$ in $H$. Clearly $V \cap A$ is $(i-1)$-dimensional. Since $A \in \mathcal{C}$, we are back to the previous case, with $A$ playing the role of $Z$.

The previous arguments now imply that all members of $\mathcal{C}$ pairwise intersect in subspaces of dimensjon $i-2$. Now suppose for two members $U, V \in \mathcal{C}$, the intersections $Z \cap U$ and $Z \cap V$ together generate $Z$. Since $Z \cap U \cap V$ has dimension $i-4$, there is a point $x \in(U \cap V)-Z$, which is then collinear to all points of $Z$, a contradiction. Hence the set $\mathcal{S}=\{Z \cap U: U \in \mathcal{C}-\{Z\}\}$ is a set of $(i-2)$-spaces of $Z$ pairwise intersecting in $(i-3)$-spaces. Dually, we have a set of lines pairwise intersecting in a point. It is easy to see that either all these lines contain a fixed point, or they are contained in a fixed plane. Hence, all members of $\mathcal{S}$ are either contained in a hyperplane $H$, or they all contain a fixed $(i-3)$-space. In the first case, by maximality of $\mathcal{C}$, all maximal singular subspaces containing $H$ belong to $\mathcal{C}$, contradicting our hypothesis on $\mathcal{C}$. Hence all members of $\mathcal{C}$ contain a fixed $(i-3)$-space $Q$.

The last case of Lemma 4.11 really occurs, for instance in the unique orthogonal polar spaces of rank 3 in projective 6-space; the maximal clique consists of all planes in a subpolar space in projective 5-space (a Klein quadric) belonging to one system of generators.

If $\Omega$ is a hyperbolic quadric, then we are also interested in the maximal cliques of the subgraph $\Delta_{i}^{*}$ of $\Delta_{i}^{\prime \prime}$, with $i=r-1$, consisting of all maximal singular subspaces belonging to one fixed system of generators, where $i$ is odd (if $i$ is even, then in the building 
$\mathcal{B}$ corresponding to the oriflamme complex, no type corresponding to generators is selfopposite). Since maximal cliques of an induced subgraph are contained in maximal cliques of the ambient graph, we immediately obtain the following corollary.

Corollary 4.12 Any maximal clique in $\Delta_{i}^{*}$, with $i=r-1$ odd, corresponds either to the set of maximal singular subspaces containing a fixed singular subspace of dimension $i-3$, or to the set of all maximal singular subspaces intersecting a fixed maximal singular subspace not belonging to $\Delta_{i}^{*}$ in a singular subspace of dimension $i-1$.

Now we handle the case of non-maximal singular subspaces.

Lemma 4.13 Any maximal clique in $\Delta_{i}^{\prime \prime}$, with $i<r-1$, corresponds either to the set of singular subspaces containing a fixed singular subspace of dimension $i-1$, or to the set of all maximal singular subspaces contained in a fixed singular subspace of dimension $i+1$.

Proof. This follows immediately from the obvious fact that, whenever three subspaces $U, V, W$ of dimension $i$ are pairwise adjacent in $\Delta_{i}^{\prime \prime}$, but do not contain a common subspace of dimension $i-1$, then they are contained in a common subspace $Z$ of dimension $i+1$, which is spanned by any pair of $\{U, V, W\}$.

Now we return to the graph $\Delta_{i}$, and assume $i>0$. It is straightforward to see that, if we endow any maximal clique of $\Delta_{i}$ with all geometric lines contained in it, then we obtain a projective space, which we refer to as a maximal geometric projective space. We sometimes omit the adjective "maximal", and refer to any subspace of a maximal geometric projective space as a geometric (projective) subspace. We easily obtain the following cases.

(I) If $\Omega$ is non-thick, and $i=r-1$, then "opposition" defines two nontrivial connected components if and only if $r$ is even, and $r$ is odd if and only if "opposition" defines a bipartite graph. Moreover, if "opposition" defines either two nontrivial connected components or a bipartite graph, then it follows that $\Omega$ is non-thick.

(II) If $\Omega$ is thick (hence not isomorphic to the polar space arising from a hyperbolic quadric), not isomorphic to a parabolic polar space, and if $i=r-1$, then every maximal clique of $\Delta_{i}$ coincides with a geometric line, which in this case is always a Grassmannian line. Hence every geometric projective space has dimension 1.

(III) If $\Omega$ is not isomorphic to a symplectic polar space, and if $i=r-2$, then every geometric projective space has dimension $i+1 \geq 2$. Moreover, every geometric line is contained in a unique geometric projective space (hence geometric projective spaces intersect in the empty set, or in a singleton).

(IV) If $\Omega$ is not isomorphic to a symplectic polar space, and if $i<r-2$, then every geometric projective space has dimension either $i+1 \geq 2$ or $r-i-1 \geq 2$. Two latter such spaces can intersect in geometric subspaces of dimension $r-i-2$ and any smaller dimension. Neither the relation "intersecting in a geometric line" nor "intersecting in a subplane" defines a bipartite graph in the set of geometric projective spaces. 
Note that, if we consider two vertices $U, V$ of $\Delta_{i}$ at distance 2 from each other, and we consider the geometry $G$ of common neighbours in $\Delta_{i}$ endowed with the geometric lines, then there are generically three possibilities: (1) $G$ consists of a single point (if $U \cap V$ has dimension $i-2$ and $U, V$ are not contained in a common singular subspace), (2) $G$ is a polar space isomorphic to the residue in $\Omega$ of any member of $Y_{i}$ (if $U \cap V$ has dimension $i-1$ and $U, V$ are not contained in a common ( $i+1$ )-dimensional subspace), (3) $G$ is a grid (if $U \cap V$ has dimension $i-2$ and $U, V$ are contained in a common singular subspace).

(V) If $\Omega$ is isomorphic to a symplectic polar space, and if $i \leq r-2$, then every geometric projective space has dimension either $i+1 \geq 2$ or $2 r-2 i-1 \geq 3$. Two such spaces can intersect in either the empty set, or a singleton, or a geometric line. The relation "intersecting in a geometric line" defines a connected bipartite graph in the set of geometric projective spaces, where two geometric projective spaces belong to the same bipartition class only if they have the same dimension.

(VI) If $\mathcal{B}$ is the oriflamme complex of a hyperbolic quadric $\Omega$, and $i=r-1$, with $r>4$ even (hence we consider one system of generators of $\Omega$ ), then the geometric projective spaces have dimension either 3 or $r-1>3$ (and $r-1$ is odd). Here, two geometric 3-spaces intersect either in the empty set, or in a geometric line, or in a singleton. Also, two geometric $(r-1)$-spaces intersect either in the empty set, or in a geometric line, but never in a singleton. The relation "intersecting in a subplane" defines a connected bipartite graph in the set of geometric projective spaces, where two geometric projective spaces belong to the same bipartition class if and only if they have the same dimension.

(VII) If $\Omega$ is isomorphic to a parabolic polar space, and if $i=r-1$, with $r$ even (note that $r$ odd is excluded from the hypothesis of Theorem 4.1), then the geometric projective spaces have dimension either 3 or $r$ (and $r$ is even). Here, as in the previous case, two geometric 3-spaces intersect either in the empty set, or in a geometric line, or in a singleton. Also, two geometric $r$-spaces intersect either in the empty set, or in a geometric line, but never in a singleton. The relation "intersecting in a geometric subspace of dimension 2 (a geometric plane)" defines a connected bipartite graph in the set of geometric projective spaces, where two geometric projective spaces belong to the same bipartition class if and only if they have the same dimension.

Consider two opposite elements of $Y_{i}$. In the graph $\Delta_{i}$, these elements are at distance $r / 2$, and it is easy to see that in no minimal path from $U$ to $V$ in $\Delta_{i}$, two adjacent vertices are contained in a Grassmannian line.

The last case needs some explanation. In particular, it might not be completely clear why the maximal clique of $\Delta_{r-1}^{\prime \prime}$ consisting of a fixed maximal subspace $V$ and all maximal subspaces meeting $V$ in a subspace of dimension $r-2$ gives rise to a geometric projective space of dimension $r$. Well, this is due to the fact that, in the ambient projective space $\mathrm{PG}(2 r, \mathbb{K})$ every $r$-space through $V$ is either tangent (and there is exactly one such) or contains a unique maximal singular subspace other than $V$. Now the reader can check that $(r+1)$-spaces through $V$ correspond to geometric lines. 
We need one more lemma before we can explain the algorithm.

Lemma 4.14 If non-opposition in the set of singular subspaces of dimension $i$ in the polar space $\Omega$ with thick lines, or in the set of generators of one self-opposite type in the oriflamme complex of a hyperbolic quadric, defines collinearity of some possible different polar space $\Omega^{\prime}$, then either $i=0$, and $\Omega=\Omega^{\prime}$, or $i=2$ and $\Omega$ is a parabolic polar space of rank 3 and $\Omega^{\prime}$ is a hyperbolic quadric in 7-dimensional projective space, or $i=3, \Omega$ is a hyperbolic quadric in 7-dimensional projective space, we are considering the set of generators of a single class of generators, and $\Omega$ is isomorphic to $\Omega^{\prime}$.

Proof. First note that every pair of collinear points in $\Omega^{\prime}$ is contained in a unique (geometric) line. But if $0<i<r-1$, then there exist non-opposite disjoint singular subspaces of dimension $i$, which are never contained in a geometric line since, by Lemma 4.7, they are not contained in a round-up triple. Therefore $i=0$ or $i=r-1$.

If $i=0$, then clearly the geometric lines containing non-opposite elements are precisely the lines of the polar space, and hence $\Omega^{\prime}=\Omega$.

Suppose now $i=r-1$. If $\Omega$ is thick and has rank $r \geq 4$, then, by Lemma 4.6, two maximal singular subspaces intersecting in a point can not be contained in a round-up triple, and hence neither in a geometric line. If $r=3$, then Lemma 4.9 implies that $\Omega$ is a parabolic polar space of rank 3. Including $\Omega$ into a hyperbolic quadric $\Omega^{\prime}$ in 7-dimensional projective space and attaching to each maximal singular subspace of $\Omega$ the unique maximal singular subspace of $\Omega^{\prime}$ belonging to one fixed system of generators, the assertion follows.

Finally suppose $i=r-1$ and $\Omega$ non-thick. Then the building $\mathcal{B}$ is the oriflamme complex and $r$ is even since otherwise either the type under consideration is not self-opposite $(r$ odd, $\mathcal{B}$ the oriflamme complex), or opposition defines a bipartite graph $(r$ odd, $\mathcal{B}$ the corresponding non-thick building), or opposition defines a non-connected graph ( $r$ even, $\mathcal{B}$ the corresponding non-thick building), a contradiction. If $r=4$, then triality implies the assertion. If $r \geq 6$, then two maximal singular subspaces intersecting in a line are never contained in a round-up triple, by Lemma 4.6.

The lemma is proved completely.

\subsection{The algorithm}

We can now put our algorithm in action. We have been given a set $Y_{i}$ of objects, together with a set $P_{i}$ of pairs of these, and the objects represent the set of vertices of some unknown self-opposite type (and of dimension $i$ ) of some unknown building $\mathcal{B}$ of rank $r$ corresponding to a polar space $\Omega$ or the oriflamme complex of a hyperbolic quadric $\Omega$. It is assumed that $\Omega$ is not a parabolic building of odd rank in case the elements of $Y_{i}$ are the maximal singular subspaces.

We can determine the geometric lines, define the graph $\Delta_{i}$ (this includes $\Delta_{i}^{*}$, which we from now on also denote by $\Delta_{i}$, since there is no confusion possibe) and determine the (maximal) geometric projective spaces. 
We run through the following steps, where it is assumed that, if we reach a certain step, then this means that the conditions of all previous steps are not satisfied. It should also be noted that each time we rerun the algorithm, we remember the inclusions of the elements of the sets $Y_{i}$ into the elements of the new set $Y_{i-1}$ or $Y_{i-2}$. This is necessary to reconstruct the precise mutual positions of the elements of $Y_{i}$ at the end. Indeed, this way we know precisely which points are incident with which elements of $Y_{i}$, as we always finish with $Y_{0}$, except in the exceptional cases of $\mathrm{D}_{4}$.

STEP 0. If the complement of the relation $P_{i}$ in $Y_{i}$ defines collinearity in some polar space, then by Lemma 4.14, we can reconstruct $\mathcal{B}$ (we cannot reconstruct the polar space, if $\mathcal{B}$ has type $D_{4}$, because we cannot distinguish between points and the two families of generators). Note that, by assumption, we cannot have a parabolic polar space of rank 3 .

STEP 1. If $P_{i}$ defines a bipartite graph in $Y_{i}$, then we know by (I) that $\Omega$ is non-thick of odd rank $r$, and $i=r-1$. The maximal distance in the graph $\Delta_{i}$ is $(r-1) / 2$. We consider the set $Y_{i-2}$ of all geometric lines and call two of them opposite if every element of one geometric line is at maximal distance of every element of the other geometric line in $\Delta_{i}$. We now run the algorithm again.

STEP 2. If $P_{i}$ has two nontrivial connected components, then we know by (I) that $\Omega$ is non-thick of even rank $r$, and $i=r-1$. In this case, we just take one of these components, and run the algorithm again. At the end we will have to remember to take the non-thick building associated to the non-thick polar space instead of the oriflamme complex.

STEP 3. If every maximal clique of $\Delta_{i}$ coincides with a geometric line, then we know, by Case (II), that we are dealing with a thick polar space not isomorphic to a parabolic polar space. Also, $i=r-1$. Consider the set of geometric lines, and define two such to be opposite if the complement of the relation $P_{i}$ in $Y_{i}$ defines a bijection between them. Then it is clear that we obtain the opposition relation in the set of singular subspaces of dimension $r-2$. We now run the algorithm again using this set and that relation.

STEP 4. If every geometric line is contained in a unique geometric subspace of dimension at least 2 , then we are in case (III). We define a new graph $\Delta_{i}^{\prime \prime}$, with vertex set $Y_{i}$. Two vertices are adjacent in $\Delta_{i}^{\prime \prime}$ if either the corresponding $i$-spaces are adjacent in $\Delta_{i}$, or if they are at distance 2 from each other in $\Delta_{i}$ and they have at least three common neighbours. It is easy to see that in the polar space $\Omega$, adjacency coincides with intersecting in a singular subspace of dimension $i-1$. Hence our notation agrees with the former use of $\Delta_{i}^{\prime \prime}$. Now Lemma 4.13 tells us that there are maximal cliques of $\Delta_{i}^{\prime \prime}$ which are maximal cliques of $\Delta_{i}$, but there are also new maximal cliques (and these correspond to subspaces $W$ of dimension $i-1$; we denote the set of those cliques by $Y_{i-1}$ ). If we endow a new maximal clique with the geometric lines, then we obtain a generalized quadrangle isomorphic to $\operatorname{Res}_{\Omega} W$. We define two of those new maximal cliques to be opposite if the relation $P_{i}$ in $Y_{i}$ restricted to these cliques does not vanish in any element. Now we run the algorithm again for these maximal cliques and the opposition relation just defined.

STEP 5. If neither "intersecting in a geometric line" nor "intersecting in a subplane" defines a bipartite graph in the set of (maximal) geometric projective spaces, then we are in Case (IV). If there exist two vertices of $\Delta_{i}$ at distance 2 such that the geometry of 
common neighbours in $\Delta_{i}$ (endowed with the geometric lines) is not a grid, then we define the graph $\Delta_{i}^{\prime \prime}$ on $Y_{i}$ as follows: two vertices are adjacent in $\Delta_{i}^{\prime \prime}$ if they are either adjacent in $\Delta_{i}$, or if they are at distance 2 in $\Delta_{i}$ and the geometry of common neighbours in $\Delta_{i}$ (endowed with the geometric lines) is neither a grid nor a single point. By Lemma 4.13, maximal cliques of $\Delta_{i}^{\prime \prime}$ that are not cliques of $\Delta_{i}$ correspond to all singular subspaces of dimension $i$ containing a fixed subspace of dimension $i-1$. We denote the set of these maximal cliques by $Y_{i-1}$. Now we again define two members of $Y_{i-1}$ to be opposite if the relation $P_{i}$ in $Y_{i}$ restricted to these cliques does not vanish in any element. We run the algorithm again for these maximal cliques and the opposition relation just defined.

Now suppose that for every pair of vertices of $\Delta_{i}$ at distance 2 admitting at least two common neighbours, the geometry of common neighbours in $\Delta_{i}$ (endowed with the geometric lines) is a grid. By case (IV) above, we know that $\Omega$ is a building of type $\mathrm{D}_{4}$. Clearly, "intersecting in a geometric line" defines a tripartite graph in the set of all geometric planes. If this is the second time the algorithm runs, then we know that in the previous time, the set $Y_{2}$ was the set of planes of $\Omega$, viewed as a polar space. Then the elements of $Y_{1}$ incident with any plane form a generic member of one class $C$ of these geometric planes. If we run the algorithm for the first time, then we know that we will not be able to distinguish the types corresponding with the end vertices of the diagram $\mathrm{D}_{4}$, and so we choose one class $C$ of the tripartite graph arbitrarily (and think of this class as the set of planes of $\Omega$ ).

We now define a new graph $\Gamma_{C}$ with vertex set $C$, where two geometric planes are adjacent if their intersection is nonempty. Consider three such planes $\pi_{1}, \pi_{2}, \pi_{3}$ pairwise adjacent but not containing a common element. Then there is a unique maximal clique of $\Gamma_{C}$ containing $\pi_{1}, \pi_{2}, \pi_{3}$ (these cliques correspond with the 3-spaces of $\Omega$ if $C$ is the set of planes). "Intersecting in a vertex" defines a bipartite graph on these maximal cliques (each bipartition class corresponds to a class of maximal singular subspaces of $\Omega$ ). Consider one such class $K$. A member $L$ of $Y_{i}$ is called incident with a member $k$ of $K$ if $L$ belongs to at least one of the geometric planes contained in $k$. We then obtain the point-line structure of $\Omega$, and have reconstructed $\Omega$ up to triality and duality, or only up to duality if we run the algorithm for the second time. Hence we have reconstructed $\mathcal{B}$ unambiguously.

STEP 6. If "intersecting in a geometric line" defines a connected bipartite graph in the set of (maximal) geometric projective spaces, then we are in Case (V). We now consider the set $Y_{i-1}$ of maximal geometric subspaces which contain geometric lines that are not the intersection of two maximal geometric subspaces. We call two members of $Y_{i-1}$ opposite if the relation $P_{i}$ in $Y_{i}$ restricted to these subspaces does not vanish in any element. We run the algorithm again for these maximal cliques and the opposition relation just defined.

STEP 7. If "intersecting in a subplane" defines a connected bipartite graph in the set of (maximal) geometric projective spaces, then we are in Cases (VI) or (VII), and some geometric projective spaces have dimension 3. There are two cases.

- If the dimension of the other geometric subspaces is odd, then we are in Case (VI). We consider the family of maximal geometric subspaces of dimension 3 and define two of those to be opposite if the relation $P_{i}$ in $Y_{i}$ restricted to these spaces does not vanish in any element. We run the algorithm again for this family and the opposition relation just 
defined.

- If the dimension of the other geometric subspaces is even, then we are in Case (VII). We remove from $\Delta_{i}$ all adjacencies occurring in any minimal path between any two opposite elements of $Y_{i}$, with here $i=r-1$ odd. The maximal cliques of the resulting graph are geometric lines corresponding to the singular subspaces of dimension $i-1$. We now proceed as in STEP 3

This completes the proof of Theorem 4.1

\section{$5 \quad$ Strong parapolar spaces arising from spherical build- ings}

In this section we look at some strong parapolar spaces arising from spherical buildings. Remember that, under Hypotesis (A), and in view of Section 4, we are primaryly concerned with the Grassmann geometries $A_{2 n-1, n}$ and $E_{7,7}$, but we will also include the Grassmann geometries $\mathrm{A}_{n, k}$ and $\mathrm{E}_{6,1}$, for $1 \leq k \leq n$, and show the following theorem.

Theorem 5.1 Let $\mathcal{B}$ be a building of type $\mathrm{A}_{n}, n>1, \mathrm{D}_{m}, m \geq 5$ and odd, or $\mathrm{E}_{6}$, and let $Y_{i}$ be the set of all vertices of certain type $i$ of $\mathcal{B}$, where $0 \leq i \leq n$ for $\mathrm{A}_{n}, i \in\left\{m-1,(m-1)^{\prime}\right\}$ for $\mathrm{D}_{m}$, or $i \in\{1,6\}$ for $\mathrm{E}_{6}$. Let $j$ be the type opposite $i$ and let $Y_{j}$ be the set of vertices of type $j$. Then the family $\mathcal{F}^{\prime}$ of subsets $\left\{v \in Y_{i}: v\right.$ is opposite $\left.w\right\}$, for $w$ running through $Y_{j}$ uniquely determines $\mathcal{B}$.

With the notation of the Theorem, and in accordance to the notation used in Subsection 3.2 under Hypothesis $\left(\mathrm{A}^{*}\right), \mathcal{H}_{\mathcal{B}, I^{\prime}}$ is the family of complements of $\mathcal{F}^{\prime}$, and $\overline{\mathcal{H}}_{\mathcal{B}, I^{\prime}}$ is the family of subsets of $Y_{i}$ obtained by intersecting arbitrary members of $\mathcal{H}_{\mathcal{B}, I^{\prime}}$, as long as the intersection contains at least two elements.

For $\mathrm{A}_{n}$ and $\mathrm{E}_{6}$, it suffices to reconstruct the Grassmann geometries, i.e., it suffices to show that the $\overline{\mathcal{H}}_{\mathcal{B}, I^{\prime}}$-round-up triples are collinear triples. For $\mathrm{D}_{m}$, we reduce to Section 4 .

We begin with $\mathrm{E}_{6}$. Since the Grassmann geometry is here a strong parapolar space of diameter 2, we might as well phrase and prove our result in these terms.

\subsection{Cases $E_{6}$ and $E_{7}$}

Theorem 5.2 Suppose that Hypothesis (A*) holds. Suppose further that the geometry $\Gamma$ satisfies the following conditions.

(i) $\Gamma$ is a strong parapolar space of point diameter 2 or 3.

(ii) $\Gamma$ has thick lines.

(iii) The symplecta of $\Gamma$ are point shadows of residues of $\mathcal{B}$.

(iv) The symplecta of $\Gamma$ are polar spaces having no round-up triples that are cocliques of their point-collinearity graphs. 
Then, with the above notation, the minimal elements of the poset $\left(\overline{\mathcal{H}}_{\mathcal{B}, I^{\prime}}, \subseteq\right)$ containing at least three points are (1) the lines of $\Gamma$ and possibly (2) cocliques of $\Delta$ consisting of points at mutual distance 3 .

Proof. First, we show that the hypothesis of Theorem 3.1 holds. for $\overline{\mathcal{H}}_{\mathcal{B}, I^{\prime}}$ in the role of $\mathcal{F}$. Indeed, by $(i i), \Gamma$ has thick lines. Conditions $(\mathrm{F} 1)$ and $(\mathrm{F} 2)$ are satisfied by the defintion of $\overline{\mathcal{H}}_{\mathcal{B}, I^{\prime}}$. By Corollary 3.9 we have $\mathcal{L} \subseteq \overline{\mathcal{H}}_{\mathcal{B}, I^{\prime}}$, therefore (F3) holds.

Now we claim that possibility (2) of the conclusion of Theorem 3.1 cannot occur for points at distance 2. Indeed, suppose $T=\{p, q, r\}$ is a round-up triple of $\Gamma$ consisting of points at mutual distance 2. Since by $(i), \Gamma$ is a strong parapolar space, there is a symplecton $S$ of $\Gamma$ containing $p$ and $q$. By (iii) and Corollary 3.10 $r \in S$, and by Proposition $3.11 T$ is a round-up triple of the point-line space induced in $\Gamma$ on $S$. This is a contradiction with Condition (iv).

Remark 5.3 All symplecta of a $J$-Grassmann geometry of a building $\mathcal{B}$ are point shadows of residues of $\mathcal{B}$.

We now show that in the case $E_{7,7}$, there are no round-up triples consisting of mutually opposite points. This will follow from the lemma below. We recall that strongly gated subgraphs were defined in Section 3.2.

Lemma 5.4 Let $\Gamma=(\mathcal{P}, \mathcal{L})$ be the J-Grassmann geometry $\mathrm{E}_{7,7}$ with point-collinearity graph $\Delta$. Suppose that $\{p, q, r\} \subseteq \mathcal{P}$ are such that $\mathrm{d}_{\Delta}(p, q)=\mathrm{d}_{\Delta}(p, r)=\mathrm{d}_{\Delta}(q, r)=3$. Then there exists a point $t \in \mathcal{P}$ such that $\left|\Delta_{2}^{*}(t) \cap\{p, q, r\}\right|=2$.

Proof. Let $S$ be a symplecton of $\Gamma$ containing $p$. By Theorem 20 of [7] $S$ is strongly gated in $\Delta$ with respect to $q$ and $r$ with gates $\{a\}=q^{\perp} \cap S$ and $\{b\}=r^{\perp} \cap S$. Since $\mathrm{d}_{\Delta}(q, r)=3$, $a \neq b$. Since $S$ is a nondegenerate polar space, there exists a point $t \in S \cap\left(a^{\perp}-b^{\perp}\right)$. Then $\{p, q\} \subseteq \Delta_{2}^{*}(t)$ and $r \notin \Delta_{2}^{*}(t)$.

\subsection{Case $\mathrm{A}_{n}$}

Let $0 \leq i \leq n-1$ and let $Y_{i}$ be the set of all $i$-dimensional subspaces of a projective space $\mathbb{P}_{n}$ of dimension $n$. By symmetry we may assume that $i \leq n / 2$. Let $\{U, V, W\}$ be a round-up triple in $Y_{i}$. Let $L$ be a line of $\mathbb{P}_{n}$ intersecting $U$ and $V$. If $L$ does not intersect $W$, then there exists an $(n-i-1)$-dimensional subspace $W^{\prime}$ through $L$ not meeting $W$, a contradiction. Hence every line meeting both $U$ and $V$ meets $W$. This implies easily that $U \cap V=V \cap W=W \cap U$ and that $W$ is a hyperplane in the subspace generated by $U$ and $V$. This yields that $U, V, W$ are contained in a line of the corresponding Grassmann geometry. 


\subsection{Case $\mathrm{D}_{m}$}

Here, with the above notation, we can identify the set $Y_{(m-1)^{\prime}}$ with the family $\mathcal{F}^{\prime}$. Hence we know all pairs of opposite elements of type $m-1$ of a non-thick polar space of rank $m$ and we are reduced to Theorem 4.1.

\section{Parapolar spaces that are not strong parapolar, arising from spherical buildings}

\subsection{Hexagonic geometries}

We call a point-line space $\Gamma=(\mathcal{P}, \mathcal{L})$ a hexagonic geometry if it satisfies the following axioms.

(H0) $\Gamma$ is a parapolar space of symplectic rank at least three.

(H1) For every point $p \in \mathcal{P}$ and every symplecton $S$ of $\Gamma, p^{\perp} \cap S$ is either empty or contains a line (equivalently, $p^{\perp} \cap S$ is never a single point).

(H2) For every point residue $\Gamma_{p}=\left(\mathcal{L}_{p}, \Pi_{p}\right)$ and every point $x \in \mathcal{L}_{p}$, the set $\{y \in$ $\left.\mathcal{L}_{p} \mid \mathrm{d}_{\Delta_{p}}(x, y) \leq 2\right\}$ is a subspace of $\Gamma_{p}$ that meets every line of $\mathcal{L}_{p}$ non-trivially.

(H3) For every point residue $\Gamma_{p}=\left(\mathcal{L}_{p}, \Pi_{p}\right)$ and every point $x \in \mathcal{L}_{p}$, there is a point $y \in \mathcal{L}_{p}$ such that $\mathrm{d}_{\Delta_{p}}(x, y)=3$.

Let $\Gamma=(\mathcal{P}, \mathcal{L})$ be a hexagonic geometry with thick lines. Suppose that $\Gamma$ satisfies one additional requirement - either some symplecton has rank exactly three or $\Gamma$ has finite singular rank. Then it was shown in [7] that $\Gamma$ is one of the following geometries: $E_{6,2}$, $\mathrm{E}_{7,1}, \mathrm{E}_{8,8}$, a metasymplectic space, or the polar Grassmannians of lines of a nondegenerate polar space of possibly infinite rank at least 4 (the labelling of the nodes of the diagrams is as in [4]). It follows that all hexagonic geometries of finite singular rank arise from spherical buildings in the situation of hypothesis (A).

In this section, we will prove the following theorems and corollaries.

Theorem 6.1 Let $\Gamma=(\mathcal{P}, \mathcal{L})$ be a hexagonic geometry with point-collinearity graph $\Delta$. Suppose that $(i) \Gamma$ is a J-Grassmann geometry of a spherical building, that is, Hypothesis (A) holds, and (ii) $\Gamma$ has thick lines. Let $\mathcal{F}_{\Delta, 2}$ be the set of all subsets of $\mathcal{P}$ that contain at least two points and are of the form $\cap\left\{\Delta_{2}^{*}(p) \mid p \in \mathcal{P}^{\prime}\right\}, \mathcal{P}^{\prime} \subseteq \mathcal{P}$. Then the minimal elements of the poset $\left(\mathcal{F}_{\Delta, 2}, \subseteq\right)$ containing at least three points are $(1)$ the lines of $\Gamma$, and (2) cocliques $C$ of $\Delta$ contained in a symplecton, such that any triple of distinct points of $C$ is a round-up triple of that symplecton.

Corollary 6.2 Suppose that the hypothesis of Theorem 6.1 holds. Then the lines of $\Gamma$ can be reconstructed from the distance three graph of $\Delta$. 
Remark 6.3 In Theorem 6.1 and Corollary 6.2 requirement $(i)$ can be dropped at the expence of lengthening the proof. See Remark 6.24 for details.

Theorem 6.4 Let $\Gamma=(\mathcal{P}, \mathcal{L})$ be a hexagonic geometry. Suppose that $\Gamma$ has thick lines. Let $\Omega=(\mathcal{P}, E)$ be the graph with the edge set $E=\left\{\{p, q\} \mid p, q \in \mathcal{P}\right.$ and $p \in q^{\perp}$ or $\{p, q\}$ is a symplectic pair $\}$. Let $\mathcal{F}_{\Omega}$ be the set of all subsets of $\mathcal{P}$ that contain at least two points and are of the form $\cap\left\{\Omega_{1}^{*}(p) \mid p \in \mathcal{P}^{\prime}\right\}, \mathcal{P}^{\prime} \subseteq \mathcal{P}$. Then the minimal elements of the poset $\left(\mathcal{F}_{\Omega}, \subseteq\right)$ containing at least three points are (1) the lines of $\Gamma$, and (2) cocliques $C$ of $\Delta$ contained in a symplecton, such that any triple of distinct points of $C$ is a round-up triple of that symplecton.

Corollary 6.5 Suppose that the hypothesis of Theorem 6.4 holds. Then the lines of $\Gamma$ can be reconstructed from the graph $\Omega$.

\subsection{Properties of hexagonic geometries}

In this section we state properties of hexagonic geometries that are needed in the proofs of Theorems 6.1 and 6.4 .

Axioms (H0) and (H1) imply that the point residues of $\Gamma$ are strong parapolar spaces and are connected. Axioms (H2) and (H3) together are equivalent to requiring that, for every point residue $\Gamma_{p}=\left(\mathcal{L}_{p}, \Pi_{p}\right)$ and every point $x \in \mathcal{L}_{p}$, the set $\left\{q \in \mathcal{L}_{p} \mid \mathrm{d}_{\Delta_{p}}(x, q) \leq 2\right\}$ is a geometric hyperplane of $\Gamma_{p}$.

Lemma 6.6 Suppose $\Gamma=(\mathcal{P}, \mathcal{L})$ is a hexagonic geometry. Let $p, x$, and $y$ be as in the axiom (H3). Let $q, r \in p^{\perp}$ be such that $x=\langle p, q\rangle$ and $y=\langle p, r\rangle$. Then $\{q, r\}$ is a special pair.

Proof. Suppose $\{q, r\}$ is a symplectic pair. By (H0) $\Gamma$ has symplectic rank at least 3, therefore there exists a line $L$ of $\Gamma$ on $p$ such that $L \subseteq q^{\perp} \cap r^{\perp}$, contradicting $\mathrm{d}_{\Delta_{p}}(x, y)=3$.

Lemma 6.7 (Theorem 21 of [7]; see also [6]) Suppose $\Gamma=(\mathcal{P}, \mathcal{L})$ is a hexagonic geometry with point-collinearity graph $\Delta$. Then the following statements hold.

(H4) For every point $p \in \mathcal{P}$, every geodesic of length 2 in $\Delta_{p}$ is extendable to a geodesic of length 3 .

(H5) For every point $p \in \mathcal{P}$, the set $\Delta_{2}^{*}(p)=\left\{q \in \mathcal{P} \mid \mathrm{d}_{\Delta}(p, q) \leq 2\right\}$ is a geometric hyperplane of $\Gamma$.

It is immediate from (H5) that the point-collinearity graph of a hexagonic geometry has diameter 3 .

Lemma 6.8 Let $\Gamma=(\mathcal{P}, \mathcal{L})$ be a hexagonic geometry. Let $S$ be a symplecton of $\Gamma$ and suppose that $p \in \mathcal{P}-S$ is such that $p^{\perp} \cap S \neq \emptyset$. Then $S \subseteq \Delta_{2}^{*}(p)$. 
Proof. Let $q \in S$. By the axiom (H1) $p^{\perp} \cap S$ contains a line $L$ of $S$. Since $S$ is a polar space, $q^{\perp} \cap L \neq \emptyset$. Therefore $q^{\perp} \cap p^{\perp} \neq \emptyset$ and $q \in \Delta_{2}^{*}(p)$.

Lemma 6.9 (Theorem 22 of [7]) Let $\Gamma=(\mathcal{P}, \mathcal{L})$ be a hexagonic geometry. Let $p \in \mathcal{P}$, and let $\Sigma=\Gamma_{p}$ be the point residue of $\Gamma$ at $p$. Then $\Sigma$ is a strong parapolar space and every plane of $\Sigma$ is contained in a symplecton of $\Sigma$.

Lemma 6.10 (Theorem 25 of [7]) Let $\Gamma=(\mathcal{P}, \mathcal{L})$ be a hexagonic geometry. Then any two symplecta of $\Gamma$ intersecting in a line intersect at least in a plane.

The following property is the "global" analogue of the local property (H4) of Lemma 6.7.

Lemma 6.11 Let $\Gamma=(\mathcal{P}, \mathcal{L})$ be a hexagonic geometry. Suppose that $p, q \in \mathcal{P}$ are such that $\mathrm{d}_{\Delta}(p, q)=2$ and $\{p, q\}$ is a special pair. Let $\{r\}=p^{\perp} \cap q^{\perp}$. Then, for every point $s \in q^{\perp}$ such that $\{r, s\}$ is a special pair, $\mathrm{d}_{\Delta}(p, s)=3$.

Proof. By the axiom (H3) and Lemma 6.6 applied to the residue $\Gamma_{q}$, there does exist a point $s \in q^{\perp}$ such that $\mathrm{d}_{\Delta}(r, s)=2$ and $\{r, s\}$ is a special pair. It remains to show that $\mathrm{d}_{\Delta}(p, s)=3$. Since $r \neq s$, and $\{p, q\}$ is a special pair, we have $s \notin p^{\perp}$. Suppose that $\mathrm{d}_{\Delta}(p, s)=2$.

Suppose first that $\{p, s\}$ is a special pair. Let $r^{\prime} \in p^{\perp} \cap s^{\perp}$. By Lemma 3.4 of [6] applied to the special pairs $\{p, q\}$ and $\{p, s\}$, we have $r^{\prime} \in r^{\perp}$. Therefore $q, r^{\prime} \in r^{\perp} \cap s^{\perp}$. Since $r^{\prime} \in p^{\perp}$ and $q \notin p^{\perp}$, we see that $q \neq r^{\prime}$. Therefore $\{r, s\}$ is a symplectic pair, contrary to the choice of $s$.

Suppose now that $\{p, s\}$ is a symplectic pair. Let $S=\mathrm{S}(p, s)$. By the axiom (H1) $r^{\perp} \cap S$ contains a line $L$ of $S$ on $p$. Let $r^{\prime}$ be the unique point of $s^{\perp} \cap L$. As in the previous case, we have $r^{\prime} \in p^{\perp}$ and $q \notin p^{\perp}$, therefore $r^{\prime}$ and $q$ are two distinct points in $r^{\perp} \cap s^{\perp}$ contradicting the choice of $s$.

\subsection{Symplecta are intersections of $\Omega_{1}^{*}(p)$}

In this section we prove the following proposition.

Proposition 6.12 Suppose that the hypothesis of Theorem 6.4 holds. Then, for every symplecton $S$ of $\Gamma$, we have $S=\cap\left\{\Omega_{1}^{*}(p) \mid p \in \mathcal{P}\right.$ and $\left.S \subseteq \Omega_{1}^{*}(p)\right\}$.

Suppose $\Gamma=(\mathcal{P}, \mathcal{L})$ is a hexagonic geometry and let the graph $\Omega=(\mathcal{P}, E)$ be as in Theorem 6.4. For a set $S \subseteq \mathcal{P}$, we let $\mathrm{Cl}_{\Omega}(S)=\cap\left\{\Omega_{1}^{*}(p) \mid p \in \mathcal{P}\right.$ and $\left.S \subseteq \Omega_{1}^{*}(p)\right\}$. Then Proposition 6.12 can be restated as: for every symplecton $S \in \mathcal{S}, S=\mathrm{Cl}_{\Omega}(S)$.

To prove Proposition 6.12 we consider cases (S1), (S2) and (S3) below; in parenthesis we list lemmas dealing with each case. Let $S \in \mathcal{S}$ and let $p \in \mathcal{P}-S$. There are three possibilities.

(S1) $S \cap\left[\Delta_{\text {spec }}(p) \cup \Delta_{3}(p)\right] \neq \emptyset($ Lemma 6.13$)$. 
(S2) $S \subseteq p^{\perp} \cup \Delta_{\text {symp }}(p)$ and $p^{\perp} \cap S \neq \emptyset$ (Lemma 6.17).

(S3) $S \subseteq \Delta_{\text {symp }}(p)$ (Lemma 6.18).

Lemma 6.13 Let $\Gamma=(\mathcal{P}, \mathcal{L})$ be a hexagonic geometry. Suppose $S \in \mathcal{S}$ and $p \in \mathcal{P}-S$ satisfy (S1). Then $p \notin \mathrm{Cl}_{\Omega}(S)$.

Proof. Let $q \in S \cap\left[\Delta_{\text {spec }}(p) \cup \Delta_{3}(p)\right]$. Then $S \subseteq \Omega_{1}^{*}(q)$ and $p \notin \Omega_{1}^{*}(q)$.

To prove Case (S2) we need Lemmas 6.14 up to 6.16 .

Lemma 6.14 Let $\Gamma=(\mathcal{P}, \mathcal{L})$ be a hexagonic geometry. Let $S \in \mathcal{S}$, let $p \in \mathcal{P}-S$, and let $Y=p^{\perp} \cap S$. Suppose that $Y$ contains a plane of $\Gamma$. Then $S \subseteq p^{\perp} \cup \Delta_{\text {symp }}(p)$.

Proof. Let $\pi \subseteq Y$ be a plane of $S$ and let $q \in S$. Since $S$ is a polar space, $q^{\perp} \cap \pi$ contains a line $L$ of $\pi$. Then $L \subseteq p^{\perp} \cap q^{\perp}$ and, therefore, $\{p, q\}$ is a symplectic pair.

Corollary 6.15 Let $\Gamma=(\mathcal{P}, \mathcal{L})$ be a hexagonic geometry. Let $S \in \mathcal{S}$, let $p \in \mathcal{P}-S$, and let $Y=p^{\perp} \cap S$. Suppose $Y \neq \emptyset$ and suppose $S \cap \Delta_{\text {spec }}(p) \neq \emptyset$. Then $Y$ is a line of $S$.

Proof. Since $S$ is a convex subspace of $\Gamma$, the set $Y$ is a singular subspace of $S$. Since $S \cap \Delta_{\text {spec }}(p) \neq \emptyset$, by Lemma $6.14(\mathrm{i})$ the set $Y$ cannot contain a plane. Therefore, by the axiom (H1) the set $Y$ is a line of $\Gamma$.

Lemma 6.16 Let $\Gamma=(\mathcal{P}, \mathcal{L})$ be a hexagonic geometry. Let $S \in \mathcal{S}$, let $p \in \mathcal{P}-S$, and let $Y=p^{\perp} \cap S$. Suppose that $Y \neq \emptyset$ and let $s \in S \cap \Delta_{2}^{*}(p)$. Then $s \in S \cap \Delta_{\text {symp }}(p)$ if and only if $s^{\perp} \cap Y$ contains a line.

Proof. Clearly, if $s^{\perp} \cap Y$ contains a line, then the pair $\{p, s\}$ is symplectic.

Suppose that the pair $\{p, s\}$ is symplectic. By the axiom (H1) $p^{\perp} \cap S$ contains a line $L$ of $\Gamma$. Since $S$ is a polar space we have $s^{\perp} \cap L \neq \emptyset$. Let $t \in p^{\perp} \cap s^{\perp} \cap S$ and let $S^{\prime}=\mathrm{S}(p, s)$. By convexity of $S^{\prime}$, we have $t \in S^{\prime}$. Since $S^{\prime} \cap S$ contains the line $\langle s, t\rangle$, by Lemma 6.10 $S^{\prime} \cap S$ contains a plane $\pi$ on $\langle s, t\rangle$. Since $S^{\prime}$ is a polar space and $p \in S^{\prime}$, the intersection $p^{\perp} \cap \pi$ is a line $M$. We have $M \subseteq s^{\perp} \cap p^{\perp} \cap S$.

Lemma 6.17 Let $\Gamma=(\mathcal{P}, \mathcal{L})$ be a hexagonic geometry. Suppose $S \in \mathcal{S}$ and $p \in \mathcal{P}-S$ satisfy (S2). Then there exists a point $q^{\prime} \in \mathcal{P}-S$ such that $S \subseteq\left(q^{\prime}\right)^{\perp} \cup \Delta_{\text {symp }}\left(q^{\prime}\right)$ and $p \in \Delta_{\text {spec }}\left(q^{\prime}\right)$. Therefore, $p \notin \mathrm{Cl}_{\Omega}(S)$.

Proof. Let $Y=p^{\perp} \cap S$ and let $q \in Y$. Since $S$ is a nondegenerate polar space, there exists $r \in\left(q^{\perp} \cap S\right)-Y$. Let $L=\langle q, r\rangle$. Since $r \notin Y$ and by hypothesis $S \subseteq p^{\perp} \cup \Delta_{\text {symp }}(p)$, we have $r \in \Delta_{\text {symp }}(p)$ and $\mathrm{d}_{\Delta_{q}}(L,\langle p, q\rangle)=2$. Therefore, by (H4) of Lemma 6.7 there exists a line $L^{\prime}$ of $\Gamma$ on $q$ such that $L^{\prime} \subseteq L^{\perp}$ and $\mathrm{d}_{\Delta_{q}}\left(L^{\prime},\langle p, q\rangle\right)=3$.

Let $s \in L^{\prime}-\{q\}$. Then $s \in \mathcal{P}-S$ and $L \subseteq s^{\perp} \cap S$. Since $r \notin Y$, we have $L \nsubseteq Y$. The space $S$ is a nondegenerate polar space of rank at least three, therefore there is a plane $\pi$ of $S$ on $L$ such that $\pi \cap Y=\{q\}$. 
Let $S^{\prime}$ be a symplecton of $\Gamma$ containing $\pi$ and $s$; if $s \in \pi^{\perp}$ then the symplecton $S^{\prime}$ exists by Lemma 6.9. We are going to find the point $q^{\prime}$ inside $S^{\prime}$. By the axiom (H1) the intersection $p^{\perp} \cap S^{\prime}$ contains a line $M$ of $S^{\prime}$ on $q$. Since $\{p, s\}$ is a special pair, by Corollary 6.15 we have $p^{\perp} \cap S^{\prime}=M$.

We claim that $M \cap \pi=\{q\}$. Indeed, suppose $M \subseteq \pi$. Then $M \subseteq Y$, therefore $M \subseteq \pi \cap Y$. A contradiction with $\pi \cap Y=\{q\}$.

By hypothesis $\pi \subseteq S \subseteq \Delta_{\text {symp }}(p)$. Therefore by Lemma $6.16 \pi \subseteq M^{\perp}$, that is $\langle\pi, M\rangle$ is a singular subspace of $S^{\prime}$. Since $S^{\prime}$ is a nondegenerate polar space, and $M \cap \pi=\{q\}$, there is a point $q^{\prime} \in\left[S^{\prime} \cap\left(\pi^{\perp}-M^{\perp}\right)\right]$. By Lemma $6.16 q^{\prime} \in \Delta_{\text {spec }}(p)$ (in particular $q^{\prime} \notin S$ ). By Lemma $6.14 S \subseteq\left(q^{\prime}\right)^{\perp} \cup \Delta_{\text {symp }}\left(q^{\prime}\right)$.

Lemma 6.18 Let $\Gamma=(\mathcal{P}, \mathcal{L})$ be a hexagonic geometry. Suppose $S \in \mathcal{S}$ and $p \in \mathcal{P}-S$ satisfy (S3). Then there exists a point $q^{\prime} \in \mathcal{P}-S$ such that $S \subseteq\left(q^{\prime}\right)^{\perp} \cup \Delta_{\text {symp }}\left(q^{\prime}\right)$ and $p \in \Delta_{\text {spec }}\left(q^{\prime}\right)$. Therefore, $p \notin \mathrm{Cl}_{\Omega}(S)$.

Proof. As in the proof of Lemma 6.17, we construct a point $q^{\prime}$ such that $\left(q^{\prime}\right)^{\perp} \cap S$ contains a plane of $S$ and $q^{\prime} \in \Delta_{\text {spec }}(p)$.

Let $r \in S$ and let $q \in p^{\perp} \cap r^{\perp}$. By hypothesis $\{p, r\}$ is a symplectic pair. Therefore, by (H4) of Lemma 6.7 applied to the lines $\langle q, p\rangle$ and $\langle q, r\rangle$ inside $\Gamma_{q}$, there is a line $L^{\prime}$ of $\Gamma$ on $q$ such that $L^{\prime} \subseteq\langle q, r\rangle^{\perp}$ and $\mathrm{d}_{\Delta_{q}}\left(L^{\prime},\langle p, q\rangle\right)=3$.

Let $s \in L^{\prime}-\{q\}$. Then $\{p, s\}$ is a special pair. Since $r \in s^{\perp} \cap S$, by the axiom (H1) $s^{\perp} \cap S$ contains a line $L$ of $S$ on $r$. Let $S^{\prime}$ be a symplecton of $\Gamma$ containing $L^{\prime}$ and $L$; if $L \subseteq\left(L^{\prime}\right)^{\perp}$, then $S^{\prime}$ exists by Lemma 6.9. By the axiom (H1) $p^{\perp} \cap S^{\prime}$ contains a line $M$ of $S^{\prime}$ on $q$. Since $\{p, s\}$ is a special pair, by Corollary $6.15 p^{\perp} \cap S^{\prime}=M$.

By Lemma 6.10 the intersection $S^{\prime} \cap S$ contains a plane $\pi$ on $L$. Since by hypothesis $\pi \subseteq S \subseteq \Delta_{\text {symp }}(p)$, by Lemma $6.16 \pi \subseteq M^{\perp}$. By hypothesis $p^{\perp} \cap S=\emptyset$, therefore $\pi \cap M=\emptyset$ and $\langle\pi, M\rangle$ is a singular subspace of $S^{\prime}$ of projective dimension 4 .

The space $S^{\prime}$ is a nondegenerate polar space, therefore there is a point $q^{\prime} \in \pi^{\perp} \cap S^{\prime}$ such that $q^{\prime} \notin M^{\perp}$. By Lemma $6.14 S \subseteq\left(q^{\prime}\right)^{\perp} \cup \Delta_{\text {symp }}\left(q^{\prime}\right)$ and by Lemma $6.16 q^{\prime} \in \Delta_{\text {spec }}(p)$.

Proposition 6.12 is immediate from Lemmas 6.13, 6.17, and 6.18.

\section{4 $\mathcal{F}_{\Delta, 2^{2}}$-round-up triples that are cocliques of $\Delta$}

In this section we prove the following.

Proposition 6.19 Let $\Gamma=(\mathcal{P}, \mathcal{L})$ be a hexagonic geometry with point-collinearity graph $\Delta$, and let $\mathcal{F}_{\Delta, 2}$ be as in Theorem 6.1. Suppose that $\Gamma$ satisfies Hypothesis (A). Then every $\mathcal{F}_{\Delta, 2}$-round-up triple of $\Gamma$, which is a coclique of $\Delta$, is a round-up triple of a symplecton.

Our proof of Proposition 6.19 relies on the following lemma. 
Lemma 6.20 Suppose $\Gamma=(\mathcal{P}, \mathcal{L})$ is a hexagonic geometry with point-collinearity graph $\Delta$. Let $\{p, q, r\} \subseteq \mathcal{P}$ be such that $\mathrm{d}_{\Delta}(p, q)=\mathrm{d}_{\Delta}(p, r)=\mathrm{d}_{\Delta}(q, r)=3$. Suppose $\left(p, s_{1}, s_{2}, q\right)$ is a geodesic of length 3 from $p$ to $q$ such that $\mathrm{d}_{\Delta}\left(r, s_{1}\right)=\mathrm{d}_{\Delta}\left(r, s_{2}\right)=2$. Then the following statements hold.

(i) There exists a point $s \in \mathcal{P}$ such that $\{p, q\} \subseteq \Delta_{\text {symp }}(s)$ and $r \in \Delta_{\text {spec }}(s) \cup \Delta_{3}(s)$.

(ii) There exists a point $s^{\prime} \in \mathcal{P}$ such that $\{p, q\} \subseteq \Delta_{2}^{*}\left(s^{\prime}\right)$ and $r \in \Delta_{3}\left(s^{\prime}\right)$.

Proof. Statement (ii) follows from statement $(i)$ and Lemmas 6.11 and 6.8. It remains to prove $(i)$.

Let $d_{p q}=\mathrm{d}_{\Delta}(p, q), d_{q r}=\mathrm{d}_{\Delta}(q, r)$, and $d_{p r}=\mathrm{d}_{\Delta}(p, r)$. Since $d_{p r}=3$ and $d_{q r}=3$, we have $\left\{s_{1}, s_{2}\right\} \subseteq \Delta_{\text {spec }}(r)$. For $i \in\{1,2\}$, let $\left\{r_{i}\right\}=r^{\perp} \cap s_{i}^{\perp}$. We are going to choose a plane $\pi$ of $\Gamma$ on $\left\langle s_{1}, s_{2}\right\rangle$ with special properties with respect to $r_{1}$. Then, we will find the point $s$ of $(i)$ inside $\pi$.

Let $L=\left\langle s_{1}, r_{1}\right\rangle$ and let $M=\left\langle s_{1}, s_{2}\right\rangle$. To choose the plane $\pi$ we consider two cases. Suppose first that $r_{1}=r_{2}$. Then let $\pi$ be a plane of $\Gamma$ on the line $M$ such that $\pi \nsubseteq r_{1}^{\perp}$. Such a plane $\pi$ can always be found inside any symplecton of $\Gamma$ containing the plane $\left\langle r_{1}, s_{1}, s_{2}\right\rangle$. We have $L^{\perp} \cap \pi=M$.

Suppose now that $r_{1} \neq r_{2}$. The distance between $L$ and $M$ in $\Delta_{s_{1}}$ is at least 2 (in fact by Lemma 3.4 of [6] the distance is 2 , but we do not require this fact here). If the distance is 3 let $\pi$ be any plane of $\Gamma$ on $L$. Suppose that the distance is 2 and let $w=\left(L, L^{\prime}, M\right)$ be a geodesic of length 2 in $\Delta_{s_{1}}$. By (H4) of Lemma $6.7 w$ extends to a geodesic $\left(L, L^{\prime}, M, N\right)$ of length 3 in $\Delta_{s_{1}}$. Let $\pi=\langle M, N\rangle$. Then $L^{\perp} \cap \pi=\left\{s_{1}\right\} \subseteq M$.

Suppose we have chosen a plane $\pi$ as above. By (H2) there are lines $L_{p}$ and $L_{q}$ in $\pi$, on $s_{1}$ and $s_{2}$ respectively, such that $L_{p} \subseteq p^{\perp} \cup \Delta_{\text {symp }}(p)$ and $L_{q} \subseteq q^{\perp} \cup \Delta_{\text {symp }}(q)$. Let $s=L_{p} \cap L_{q}$. Then $s \in \Delta_{\text {symp }}(p) \cap \Delta_{\text {symp }}(q)$ (we have $s \notin p^{\perp} \cup q^{\perp}$, since $d_{p q}=3$ ).

We claim that $s \in \Delta_{\text {spec }}(r) \cup \Delta_{3}(r)$. Indeed, since $\mathrm{d}_{\Delta}(r, p)=3$ and $s \in \Delta_{\text {symp }}(p)$, by (H1) we have $s \notin r^{\perp}$. Suppose $s \in \Delta_{\text {symp }}(r)$. Let $S=\mathrm{S}(r, s)$. Since the pair $\left\{r, s_{1}\right\}$ is special, we have $s_{1} \notin S$. By the axiom (H1) $s_{1}^{\perp} \cap S$ contains a line $L_{1}$ of $S$ on $s$. Since $S$ is a polar space, $r^{\perp} \cap L_{1} \neq \emptyset$. Therefore, $r_{1} \in L_{1}$. This implies that $r_{1} \in s^{\perp}$ and, therefore, $s \in L^{\perp}$.

By the choice of the plane $\pi$, we have $L^{\perp} \cap \pi \subseteq M$. Therefore $s \in M$. But this is impossible, since $s_{2} \in \Delta_{\text {spec }}(p)$ and therefore $L_{p} \neq M$. This contradiction proves the claim. We have shown that $s \in\left[\Delta_{\text {symp }}(p) \cap \Delta_{\text {symp }}(q)\right] \cap\left[\Delta_{\text {spec }}(r) \cup \Delta_{3}(r)\right]$

Proof of Proposition 6.19. Let $T=\{p, q, r\}$ be a coclique of $\Delta$ of size three and suppose that $T$ is a round-up triple of $\Gamma$. Let $d_{p q}=\mathrm{d}_{\Delta}(p, q), d_{q r}=\mathrm{d}_{\Delta}(q, r)$, and $d_{p r}=\mathrm{d}_{\Delta}(p, r)$. By Corollary 3.14 we only need to consider Cases 1 up to 3 below. In Cases 1 and 2 we construct a point $x \in \mathcal{P}$, such that $\left|T \cap \Delta_{2}^{*}(x)\right|=2$.

Case 1. All distances are equal to 3.

Let $\left(p, s_{1}, s_{2}, q\right)$ be a geodesic of length 3 from $p$ to $q$ in $\Delta$. Suppose first that $\mathrm{d}_{\Delta}\left(r, s_{1}\right)=3$. Then $p, q \in \Delta_{2}^{*}\left(s_{1}\right)$ and $r \notin \Delta_{2}^{*}\left(s_{1}\right)$, and we can put $x=s$. A similar consideration applies when $\mathrm{d}_{\Delta}\left(r, s_{2}\right)=3$. Therefore we can assume that $\mathrm{d}_{\Delta}\left(r, s_{1}\right)=2$ and $\mathrm{d}_{\Delta}\left(r, s_{2}\right)=2$. This 
case is precisely Lemma 6.20(ii) and we can put $x=s^{\prime}$, where $s^{\prime}$ is as in that lemma.

Case 2. All distances are equal to 2 and all pairs are special.

Let $\{s\}=p^{\perp} \cap q^{\perp}$. We consider four subcases.

Case 2.1. The hypothesis of Case 2 holds and $\mathrm{d}_{\Delta}(r, s)=3$. Then $p, q \in \Delta_{2}^{*}(s)$ and $r \notin \Delta_{2}^{*}(s)$, and we are done.

Case 2.2. The hypothesis of Case 2 holds and $s \in \Delta_{\text {spec }}(r)$. By Lemma 6.11 there is a point $x \in s^{\perp}$ such that $\mathrm{d}_{\Delta}(r, x)=3$. Then $x$ is the required point.

Case 2.3. The hypothesis of Case 2 holds and $s \in \Delta_{\text {symp }}(r)$.

Let $S$ be a symplecton of $\Gamma$ containing $s$ and $r$ and let $\left\{r^{\prime}\right\}=p^{\perp} \cap r^{\perp}$. Since the pair $\{p, r\}$ is special, by Corollary $6.15 p^{\perp} \cap S=L$ for some line $L$ of $S$ on $s$. Since $r \in S$, we have $r^{\perp} \cap L \neq \emptyset$, therefore $r^{\prime} \in L$.

Let $S^{\prime}$ be any symplecton of $\Gamma$ containing the plane $\langle p, L\rangle$. Since $r^{\perp} \cap S^{\prime} \neq \emptyset$ and $\{p, r\}$ is a special pair, by Corollary $6.15 r^{\perp} \cap S^{\prime}=M$ for some line $M$ of $S^{\prime}$ on $r^{\prime}$. By the hypothesis of Case $2.3 s \in \Delta_{\text {symp }}(r)$, therefore by Lemma $6.16 s \in M^{\perp}$.

Let $\pi=\langle s, M\rangle$. Since $S^{\prime}$ is a nondegenerate polar space of rank at least three, considering the plane $\pi$ and the line $\langle s, p\rangle$ in the residue $\Gamma_{s}$ we see that there is a plane $\pi^{\prime}$ of $S^{\prime}$ on $\langle s, p\rangle$ such that $\left(\pi^{\prime}\right)^{\perp} \cap \pi=\{s\}$. Then $M^{\perp} \cap \pi^{\prime}=\{s\}$, therefore by Lemma 6.16 applied to $S^{\prime}$ and $r$ we obtain that $\pi^{\prime}-\{s\} \subseteq \Delta_{\text {spec }}(r)$.

By the axiom (H2) applied to $\langle q, s\rangle$ and $\pi^{\prime}$ in $\Gamma_{s}$, there is a point $s^{\prime} \in \pi^{\prime}-\{s\}$ such that the pair $\left\{q, s^{\prime}\right\}$ is symplectic. Then $s^{\prime} \in p^{\perp} \cap \Delta_{\text {symp }}(q) \cap \Delta_{\text {spec }}(r)$. By Lemma 6.11 there is a point $x \in\left(s^{\prime}\right)^{\perp}$ such that $\mathrm{d}_{\Delta}(r, x)=3$. We have $\mathrm{d}_{\Delta}(x, p) \leq 2$ and by Lemma 6.8 $\mathrm{d}_{\Delta}(x, q) \leq 2$.

Case 2.4. The hypothesis of Case 2 holds and $s \in r^{\perp}$. Let $S$ be any symplecton of $\Gamma$ on the line $\langle p, s\rangle$. By Corollary $6.15 q^{\perp} \cap S=L_{q}$ and $r^{\perp} \cap S=L_{r}$ for some lines $L_{q}$ and $L_{r}$ of $S$ on $s$.

Since $\{q, r\}$ is a special pair, we have $L_{q} \neq L_{r}$. The space $S$ is a nondegenerate polar space, therefore there exists a point $s^{\prime} \in S$ such that $s^{\prime} \in L_{q}^{\perp}-L_{r}^{\perp}$. Then by Lemma 6.16 $s^{\prime} \in\left[p^{\perp} \cup \Delta_{\text {symp }}(p)\right] \cap\left[q^{\perp} \cup \Delta_{\text {symp }}(q)\right] \cap \Delta_{\text {spec }}(r)$. The required point $x$ exists by Lemmas 6.11 and 6.8, as in Case 2.3.

Case 3. All distances are equal to 2 and all pairs are symplectic.

Let $S=\mathrm{S}(p, q)$. By Corollary $3.10 r \in S$. By Proposition 3.11, since $T$ is a round-up triple of $\Gamma$, it is a round-up triple of the symplecton $S$.

\section{5 $\mathcal{F}_{\Omega}$-round-up triples that are cocliques of $\Delta$}

Proposition 6.21 Let $\Gamma=(\mathcal{P}, \mathcal{L})$ be a hexagonic geometry with point-collinearity graph $\Delta$. Then every $\mathcal{F}_{\Omega}$-round-up triple of $\Gamma$, which is a coclique of $\Delta$, is a round-up triple of a symplecton. 
Proof. Suppose that $T=\{p, q, r\}$ is a coclique of $\Delta$ and let $d_{p q}=\mathrm{d}_{\Delta}(p, q), d_{q r}=\mathrm{d}_{\Delta}(q, r)$, and $d_{p r}=\mathrm{d}_{\Delta}(p, r)$. We consider five cases. In Cases 1-4 and in Case 5(2) we show that there exists $x \in \mathcal{P}$ such that $\left|T \cap \Omega_{1}^{*}(x)\right|=2$.

Case 1. All distances are equal to 3.

Let $s_{1}, s_{2} \in \mathcal{P}$ be such that $\left(p, s_{1}, s_{2}, q\right)$ is a walk of length 3 . Suppose first that $\mathrm{d}_{\Delta}\left(r, s_{1}\right)=$ 3 or $\mathrm{d}_{\Delta}\left(r, s_{2}\right)=3$. Let $\pi$ be a plane of $\Gamma$ on the line $\left\langle s_{1}, s_{2}\right\rangle$. By the axiom (H2) there are lines $L_{p}$ and $L_{q}$ in $\pi$, such that $p$ and $L_{p}$ lie in a symplecton of $\Gamma$, and $q$ and $L_{q}$ lie in a symplecton of $\Gamma$. Let $x=L_{p} \cap L_{q}$. Then $p, q \in \Omega_{1}^{*}(x)$. Since $\pi$ contains a point at distance 3 from $r$, by Lemma 6.8 we have $x \notin \Omega_{1}^{*}(r)$.

Suppose now that $\mathrm{d}_{\Delta}\left(r, s_{1}\right)=2$ and $\mathrm{d}_{\Delta}\left(r, s_{2}\right)=2$. Then by Lemma 6.20(i) there exists a point $x \in \mathcal{P}$ such that $x \in\left[\Delta_{\text {symp }}(p) \cap \Delta_{\text {symp }}(q)\right] \cap\left[\Delta_{\text {spec }}(r) \cup \Delta_{3}(r)\right]$

Case 2. At least one distance is 2 and at least one distance is 3.

Suppose that $d_{p q}=2$ and $d_{p r}=3$. Let $s \in p^{\perp} \cap q^{\perp}$. Then by Lemma 6.8 either $\mathrm{d}_{\Delta}(r, s)=3$, or $s \in \Delta_{\text {spec }}(r)$. Therefore we can put $x=s$.

Case 3. All distances are equal to 2 , there is at least one symplectic pair, and there is a at least one special pair.

Without loss of generality we can assume that $\{p, q\}$ is a symplectic pair and $\{p, r\}$ is a special pair. Then $p, q \in \Omega_{1}^{*}(p)$ and $r \notin \Omega_{1}^{*}(p)$. Therefore we can put $x=p$.

Case 4. All distances are equal to 2 and all pairs are special.

Let $s \in p^{\perp} \cap q^{\perp}$. There are two subcases.

Case 4.1. The hypothesis of Case 4 holds and $s \in \Delta_{\text {spec }}(r) \cup \Delta_{3}(r)$.

In this case $p, q \in s^{\perp} \subseteq \Omega_{1}^{*}(s)$ and $r \notin \Omega_{1}^{*}(s)$. Therefore we can let $x=s$.

Case 4.2. The hypothesis of Case 4 holds and $s \in r^{\perp} \cup \Delta_{\text {symp }}(r)$.

Let $S$ be any symplecton of $\Gamma$ containing the pair $\{r, s\}$. Since the pairs $\{p, r\}$ and $\{q, r\}$ are special, by Corollary $6.15 p^{\perp} \cap S=L_{p}$ and $q^{\perp} \cap S=L_{q}$ for some lines $L_{p}$ and $L_{q}$ of $S$ on $s$.

The pair $\{p, q\}$ is special, therefore $L_{p} \neq L_{q}$. Since $S$ is a nondegenerate polar space, there is a point $x \in L_{p}^{\perp}-L_{q}^{\perp}$. By Lemma $6.16 x \in p^{\perp} \cup \Delta_{\text {symp }}(p)$ and $x \notin q^{\perp} \cup \Delta_{\text {symp }}(q)$. Since $x \in r^{\perp} \cup \Delta_{\text {symp }}(r)$, we obtain that $p, r \in \Omega_{1}^{*}(x)$ and $q \notin \Omega_{1}^{*}(x)$.

Case 5. All distances are equal to 2 and all pairs are symplectic.

Let $S=\mathrm{S}(p, q)$. By Proposition 6.12 we can assume that $r \in S$. Now there are two possibilties: (1) $\{p, q, r$,$\} is a round-up triple of S,(2)$ there is a point $s \in S \cap\left(\{p, q\}^{\perp}-r^{\perp}\right)$. We show that (2) cannot occur.

Let $S^{\prime}=\{p, q\}^{\perp} \cap S$. Since $S$ is a polar space of rank at least 3, its subspace $\Gamma \mid S^{\prime}$ is a polar space of rank at least 2. Since $s \in S \cap\left(\{p, q\}^{\perp}-r^{\perp}\right)$, the set $r^{\perp} \cap S^{\prime}$ is a proper hyperplane of $S^{\prime}$. Therefore there is a line $L \subseteq\{p, q\}^{\perp}$ on $s$ such that $L \nsubseteq r^{\perp}$. Let $\Delta^{\prime}=\Delta_{s}$. Let $M$ be a line of $S$ on $s$ such that $\mathrm{d}_{\Delta^{\prime}}(M, L)=2$. By axiom (H3) applied to $\Gamma_{s}$, there is a point $x \in L^{\perp}-S$ such that $\mathrm{d}_{\Delta^{\prime}}(M,\langle s, x\rangle)=3$. By Corollary 6.15 and 
Lemma $6.16\{p, q\} \subseteq \Omega_{1}^{*}(x)$ and $r \notin \Omega_{1}^{*}(x)$.

Lemma 6.22 Let $\Gamma=(\mathcal{P}, \mathcal{L})$ be a hexagonic geometry with point-collinearity graph $\Delta$, and let the graph $\Omega$ be as in Theorem 6.4. Then, for every $p \in \mathcal{P}$, the set $\Omega_{1}^{*}(p)$ is a subspace of $\Gamma$.

Proof. Let $p \in \mathcal{P}$, let $q, r \in \Omega_{1}^{*}(p)$, and suppose that $q \in r^{\perp}-\{r\}$. Let $L=\langle q, r\rangle$. We need to show that $L \subseteq \Omega_{1}^{*}(p)$. Since $\Omega_{1}^{*}(p)=p^{\perp} \cup \Delta_{\text {symp }}(p)$, there are the following possibilities.

Case 1. $q, r \in p^{\perp}$.

Since $\Gamma$ is a parapolar space, $\Gamma$ is a gamma space. Therefore $L \subseteq p^{\perp} \subseteq \Omega_{1}^{*}(p)$.

Case 2. $q \in p^{\perp}$ and $r \notin p^{\perp}$.

Since $r \in \Omega_{1}^{*}(p)-p^{\perp}$, we have $r \in \Delta_{\text {symp }}(p)$. Let $S=\mathrm{S}(p, r)$. By convexity of $S$ we have $q \in S$. Since $S$ is a subspace of $\Gamma$, and $q, r \in L \cap S$, we obtain that $L \subseteq S \subseteq \Omega_{1}^{*}(p)$.

The case $r \in p^{\perp}$ and $q \notin p^{\perp}$ is similar to Case 2 .

Case 3. $q, r \in \Omega_{1}^{*}(p)-p^{\perp}$. In this case $q, r \in \Delta_{\text {symp }}(p)$ and there are two subcases.

Case 3.1. The hypothesis of Case 3 holds and $\mathrm{S}(p, q)=\mathrm{S}(p, r)$.

In this case $L \subseteq \mathrm{S}(p, q) \subseteq \Omega_{1}^{*}(p)$.

Case 3.2. The hypothesis of Case 3 holds and $\mathrm{S}(p, q) \neq \mathrm{S}(p, r)$.

Let $S=\mathrm{S}(p, r)$. Since $q^{\perp} \cap S \neq \emptyset$, by the axiom (H1) the intersection $q^{\perp} \cap \mathrm{S}(p, r)$ contains a line $L$ of $S$ on $r$. Since $S$ is a polar space we have $p^{\perp} \cap L \neq \emptyset$. Let $\{s\}=p^{\perp} \cap L$ and let $\pi=\langle q, r, s\rangle$ (the span of $\{q, r, s\}$ is not a line since that would imply $\mathrm{S}(p, q)=\mathrm{S}(p, r)$ ). By hypothesis of Case $3 q, r \in \Delta_{\text {symp }}(p)$ and $q \neq r$, therefore by the axiom (H2) applied to $\pi$ and $\langle p, s\rangle$ in the residue $\Gamma_{s}$ all lines of $\pi$ on $s$ are contained in $p^{\perp} \cup \Delta_{\text {symp }}(p)$. This implies $L \subseteq \pi \subseteq \Delta_{\text {symp }}(p) \subseteq \Omega_{1}^{*}(p)$.

\subsection{Proofs of Theorems 6.1 and 6.4, and Corollaries 6.2 and 6.5}

Proof of Theorem 6.1. First, we show that the hypothesis of Theorem 3.1 holds.

By hypothesis $\Gamma$ has thick lines. By (H5) of Lemma $6.7 \Delta_{2}^{*}(p)$ is a hyperplane of $\Gamma$. Therefore conditions (F1) and (F2) are satisfied by the definition of $\mathcal{F}_{\Delta, 2}$.

By Part $(i)$ of the hypothesis $\Gamma$ arises from spherical building and one can show that the hyperplanes $\Delta_{2}^{*}(p)$ are precisely the elements of $\mathcal{H}_{\mathcal{B}, I^{\prime}}$. Then Corollary 3.9 implies that $\mathcal{L} \subseteq \mathcal{F}_{\Delta, 2}$, that is (F3) holds.

Let $X \in \mathcal{F}_{\Delta, 2}$ be minimal and suppose $|X| \geq 3$. Then by Theorem $3.1 X \in \mathcal{L}$ or $\Delta \mid X$ is a coclique, and every triple of distinct points of $X$ is an $\mathcal{F}_{\Delta, 2}$-round-up triple. Suppose $\Delta \mid X$ is a coclique and let $T \subseteq X$ be a triple of distinct points. Then by Proposition 6.19 $T$ is a round-up triple of a symplecton $S \in \mathcal{S}$. Since $|X \cap S| \geq 3 \geq 2$, and by Corollary $3.9 S \in \mathcal{F}_{\Delta, 2}$, we obtain $X \subseteq S$ by the minimality of $X$. 
Proof of Corollary 6.2. Suppose $\{p, q\}$ are two distinct collinear points of $\Gamma$ and let $\{q, r\}$ be distinct collinear points of $\Gamma$ or a symplectic pair in $\Gamma$. Then by Lemma 6.8 $\mathrm{d}_{\Delta}(p, r) \leq 2$.

Suppose now that $\{p, q\}$ is a symplectic pair in $\Gamma$ and let $r \in p^{\perp} \cap q^{\perp}$. By (H4) of Lemma 6.7 applied to $\Gamma_{r}$ there exists a point $s \in r^{\perp} \cap q^{\perp}$ such that $\{p, s\}$ is a special pair. Using (H4) in $\Gamma_{s}$, we see that there exists a point $t \in s^{\perp}$ such that $\{q, t\}$ is a symplectic pair and $\{r, t\}$ is a special pair. Now by Lemma 6.11 applied to the walk $(p, r, s, t)$ we obtain that $\mathrm{d}_{\Delta}(p, t)=3$.

By Theorem 6.1 we either know all collinear pairs in $\Gamma$, or we know all collinear and symplectic pairs in $\Gamma$. In the latter case the argument above gives a way of distinguishing collinear pairs from symplectic pairs.

To prove Theorem 6.4 we need the following lemma.

Lemma 6.23 Let $\Gamma=(\mathcal{P}, \mathcal{L})$ be a hexagonic geometry with point-collinearity graph $\Delta$. Let $\mathcal{F}_{\mathcal{S}}$ be the set of all subsets of $\mathcal{P}$ that contain at least two points and are of the form $\cap\left\{S \mid S \in \mathcal{S}^{\prime}\right\}, \mathcal{S}^{\prime} \subseteq \mathcal{S}$. Then $\mathcal{L} \subseteq \mathcal{F}_{\mathcal{S}}$.

Proof. Let $L \in \mathcal{L}$ and let $\mathcal{S}_{L}$ be the set of all symplecta of $\Gamma$ containing $L$. Since $\Gamma$ is a parapolar space, by (PPS3) $\mathcal{S}_{L} \neq \emptyset$. Let $X=\cap\left\{S \mid S \in \mathcal{S}_{L}\right\}$. Clearly, $L \subseteq X$. It remains to show that $X \subseteq L$.

Let $S \in \mathcal{S}_{L}$. Let $\Pi^{\prime}$ be the set of all planes of $S$ on $L$ and let $Y=\cap\left\{\rho^{\perp} \mid \rho \in \Pi^{\prime}\right\}$. Since $S$ is a nondegenerate polar space, we have $Y=L$. Let $p \in L$. By (H0) $S$ has rank at least 3 , therefore there exists a geodesic $w=\left(L_{0}, L_{1}, L\right)$ of length 2 in $\Delta_{p}$, where $L_{0}$ and $L_{1}$ are lines of $S$. By (H4) of Lemma $6.7 w$ extends to a geodesic $\left(L_{0}, L_{1}, L, L_{3}\right)$ of length 3 . Let $\pi=\left\langle L, L_{3}\right\rangle$. Then $\pi$ is a plane of $\Gamma$ on $L$ such that $\pi \nsubseteq S$.

Let $\rho \in \Pi^{\prime}$. Using Lemma 6.9 if $\rho \subseteq \pi^{\perp}$, there is a symplecton $S_{\rho}$ containing $\pi$ and $\rho$. Let $Y_{\rho}=S \cap S_{\rho}$. Since $\pi \nsubseteq S$, we have $S \neq S_{\rho}$. Therefore the space $Y_{\rho}$ is a singular subspace of $S$ containing the plane $\rho$. This implies $Y_{\rho} \subseteq \rho^{\perp}$. Let $Y^{\prime}=\cap\left\{Y_{\rho} \mid \rho \in \Pi^{\prime}\right\}$. By the definition of $X$ we have $X \subseteq Y^{\prime}$. By the preceeding paragraph $Y^{\prime} \subseteq Y$. Therefore $X \subseteq Y^{\prime} \subseteq Y=L$.

Remark 6.24 Suppose $\Gamma=(\mathcal{P}, \mathcal{L})$ is a hexagonic geometry, not necessarily arising from a spherical building. One can show directly from the point-line axioms (using results of Section 6.3 and Lemmas 6.11, 6.14, and 6.8) that

$(*) \mathcal{F}_{\mathcal{S}} \subseteq \mathcal{F}_{\Delta, 2}$.

Therefore, by Lemma $6.23 \mathcal{L} \subseteq \mathcal{F}_{\Delta, 2}$. One can also show, considering cases omitted by Corollary 3.14, and using $(*)$ to modify the proof of Case 3, that the conclusion of Proposition 6.19 holds for all hexagonic geometries. Therefore Theorem 6.1 and Corollary 6.2 generalize to all hexagonic geometries with thick lines.

Proof of Theorem 6.4. First, we show that the hypothesis of Theorem 3.1 holds.

By hypothesis $\Gamma$ has thick lines. By Lemma 6.22 , for every $p \in \mathcal{P}$, the set $\Omega_{1}^{*}(p)$ is a 
subspace of $\Gamma$. Therefore by the definition of $\mathcal{F}_{\Omega}$ conditions (F1) and (F2) hold. By Lemma $6.23 \mathcal{L} \subseteq \mathcal{F}_{\mathcal{S}}$, and by Proposition $6.12 \mathcal{F}_{\mathcal{S}} \subseteq \mathcal{F}_{\Omega}$. Therefore (F3) holds.

Let $X \in \mathcal{F}_{\Delta, 2}$ be a minimal element and suppose $|X| \geq 3$. By Theorem $3.1 X \in \mathcal{L}$ or $\Delta \mid X$ is a coclique, and every triple of distinct points in $X$ is an $\mathcal{F}_{\Omega}$-round-up triple. Suppose $\Delta \mid X$ is a coclique and let $T \subseteq X$ be a triple of distinct points. Then by Proposition 6.21 $T$ is a round-up triple of a symplecton $S \in \mathcal{S}$. Since $|X \cap S| \geq 3 \geq 2$, and by Proposition $6.12 S \in \mathcal{F}_{\Omega}$, we obtain $X \subseteq S$ by the minimality of $X$.

Proof of Corollary 6.5. By Theorem 6.4 we either know (1) the round-up triples consisting of pairwise collinear points of $\Gamma$ or, else, we know (2) the round-up triples consisting of pairwise collinear points, together with the round-up triples consisting of points forming symplectic pairs with each other in $\Gamma$. In case (2) we need a way of distinguishing the two kinds of round-up triples. Note that we can distinguish case (1) from case (2) by looking at the diameter of the graph on the point set where adjacency is "being contained in a round-up triple" (in case (1), this diameter is 3 ; in case (2) it is 2).

Suppose case (2) occurs. Then, from the list of hexagonic geometries satsifying Hypothesis $(\mathrm{A})$, we see that $\Gamma$ must be a geometry $F_{4,1}$ such that its symplecta are unitary, symplectic or non-embeddable polar spaces, or the characteristic of the underlying field is 2 and the building of type $F_{4}$ is of mixed type. We refer to this as Hypothesis $(\mathbf{F})$.

Suppose $\Gamma$ satisfies Hypothesis (F). Let $S$ be a symplecton of $\Gamma$. For every round-up triple $T$ of $S$, let $L_{T}$ be the set consisting of all $p \in S$ for which there are $a, b \in S$ such that $\{a, b, p\}$ is a round-up triple of $S$. If $T$ consists of collinear points, then $L_{T}$ is the unique line of $\Gamma$ containing $T$. If $T$ consists of noncollinear points, then $L_{T}$ is a "hyperbolic" line of $\Gamma \mid S$. Under the assumption of Hypothesis $(\mathrm{F})$, a hyperbolic line is the set of points collinear with all points of two opposite lines of $\Gamma \mid S$. This follows from the fact that every symplecton either admits a polarized embedding in projective 5-space, or is non-embeddable.

We now show the following proposition.

Proposition 6.25 Suppose $\Gamma$ satisfies Hypothesis $(\mathrm{F})$. Let $p$ and $p^{\prime}$ be two points at distance 3. Put $\mathcal{P}_{\left\{p, p^{\prime}\right\}}=\Delta_{\text {symp }}(p) \cap \Delta_{\text {symp }}\left(p^{\prime}\right)$. Let $\mathcal{L}_{\left\{p, p^{\prime}\right\}}$ be the set of hyperbolic lines entirely contained in $\mathcal{P}_{\left\{p, p^{\prime}\right\}}$. Then $\left(\mathcal{P}_{\left\{p, p^{\prime}\right\}}, \mathcal{L}_{\left\{p, p^{\prime}\right\}}\right)$ is isomorphic to a symplecton in the $\mathrm{F}_{4,4}$ geometry dual to $\Gamma$ with respect to the underlying building.

Proof. A symplecton in the $F_{4,4}$ geometry dual to $\Gamma$ with respect to the underlying building is isomorphic to the dual geometry of a point residue in $\Gamma$. More precisely, it is isomorphic to rank 3 polar space with point set the set $\mathcal{P}_{p}$ of symplecta containing $p$, line set the set $\mathcal{L}_{p}$ of planes through $p$ and plane set the set of lines through $p$, with natural incidence. Since a polar space is determined by its points and lines, it suffices to establish a bijection $\beta: \mathcal{P}_{p} \rightarrow \mathcal{P}_{\left\{p, p^{\prime}\right\}}$ inducing a bijection $\mathcal{L}_{p} \rightarrow \mathcal{L}_{\left\{p, p^{\prime}\right\}}$.

In the sequel, we use the following well-known property of metasymplectic spaces. Given a point $x$ and a symplecton $S$ not containing $x$, then either (1) there is a unique point $x^{\prime} \in S \cap \Delta_{\text {symp }}(x)$, all points in $S$ collinear with $x^{\prime}$ belong to $\Delta_{\text {spec }}(x)$, and all other points of $S$ are at distance 3 from $x$ (and we say that $x, S$ are in relative position 1), or (2) 
there is a unique line $L$ in $S$ such that all points of $L$ are collinear with $x$, all points in $S$ collinear with all points of $L$ belong to $\Delta_{\text {symp }}(x)$ (and the symplecta through $x$ and such a point contain $L$ ), and all other points of $S$ belong to $\Delta_{\text {spec }}(x)$ (relative position 2). It follows that, if two symplecta meet in a unique point $x$, then each point opposite $x$ in one symplecton is at distance 3 in $\Gamma$ from any point opposite $x$ in the other symplecton. We will also use the fact that, if two distinct symplecta meet in at least two points, then they meet in a plane.

Let $S$ be a symplecton through $p$. Since $S$ contains a point at distance 3 from $p^{\prime}$, we see that $S, p^{\prime}$ are in relative position 1 . Hence there is a unique point $S^{\beta} \in S$ such that $\left\{p^{\prime}, S^{\beta}\right\}$ is a symplectic pair. This defines $\beta$. It is easy to see that $\beta$ is a bijection, since there is a unique symplecton through a symplectic pair. Now let $\pi$ be any plane through $p$, and choose two arbitrary symplecta $S_{1}, S_{2}$ containing $\pi$. Let $S_{1}^{\prime}, S_{2}^{\prime}$ be the symplecta containing $p^{\prime}$ and $x_{1}=: S_{1}^{\beta}, x_{2}=: S_{2}^{\beta}$, respectively. If $S_{1}^{\prime}$ and $S_{2}^{\prime}$ met in a unique point (namely, $p^{\prime}$ ), then $x_{1}$ and $x_{2}$ would be at distance 3 . But there is a path of length 2 connecting them via a point of $\pi$. Hence $S_{1}^{\prime} \cap S_{2}^{\prime}$ is a plane $\pi^{\prime}$. Now we see that there are at least two paths of length 2 connecting $x_{1}$ with $x_{2}$; one via $\pi$ and one via $\pi^{\prime}$. Hence $\left\{x_{1}, x_{2}\right\}$ is a symplectic pair. It follows from mutual position 2 that $x_{1}$ and $x_{2}$ are collinear to all points of a common line $L$ of $\pi$, and likewise of a common line $L^{\prime}$ of $\pi^{\prime}$. Now consider the symplecton $S$ containing $x_{1}$ and $x_{2}$. The hyperbolic line $H$ through $x_{1}, x_{2}$ in $S$ is precisely the set of points collinear with all points of $L \cup L^{\prime}$. Let $x_{3} \in H$ be arbitrary. Then there is plane $\pi_{3}$ containing $x_{3}$ and $L$, and a plane $\pi_{3}^{\prime}$ containing $x_{3}$ and $L^{\prime}$. The symplecta $S_{3}$ and $S_{3}^{\prime}$ defined by the planes $\pi, \pi_{3}$ and $\pi^{\prime}, \pi_{3}^{\prime}$, respectively, meet in $x_{3}$ and hence $x_{3} \in \mathcal{P}_{\left\{p, p^{\prime}\right\}}$. Conversely, let $S_{4}$ be any symplecton through $\pi$, and let $S_{4}^{\prime}$ be the symplecton defined by $p^{\prime}$ and $x_{4}=: S_{4}^{\beta}$. The three symplecta $S_{1}^{\prime}, S_{2}^{\prime}, S_{4}^{\prime}$ meet pairwise in planes, hence they share a common line $R$ (this line corresponds to a plane of the rank 3 polar space, defined by the residue in $p^{\prime}$, containing the three lines of the polar space defined by the three planes of $\Gamma$ through $p^{\prime}$ ). It follows that $R$ contains a point $y^{\prime}$ collinear with $x_{1}, x_{2}, x_{4}$. Also, since $L$ belongs to $S_{4}$, there is a point $y$ on $L$ collinear with $x_{4}$ (in fact, by the foregoing - namely, interchanging the roles of $x_{2}$ and $x_{4}$-all points of $L$ are collinear with $x_{4}$ ). Hence $x_{1}, x_{2}$ and $x_{4}$ are contained in a symplecton defined by $y, y^{\prime}$, which thus coincides with the symplecton $S$ through $x_{1}, x_{2}$. Hence, by the foregoing, $x_{4}$ belongs to $H$. It now also follows that $S_{4}^{\prime}$ contains $\pi^{\prime}$.

The proposition is proved.

Proposition 6.25 shows that, if we consider two points at distance 3 , and we consider the set of all points collinear or forming a symplectic pair with both $p, p^{\prime}$, and we endow this set with all (hyperbolic) lines - but it is easy to see that there are no ordinary lines; just hyperbolic ones - then the maximal cliques are projective planes.

Now let $\left\{p, p^{\prime}\right\}$ be a special pair, and let $x$ be the point collinear with both $p$ and $p^{\prime}$. Let $S$ be any symplecton through $p$ and $x$. Then there is a line $M$ in $S$ each point of which is collinear with $p^{\prime}$. Let $\pi$ be a plane through $M$ in $S$. Each point of $\pi$ forms a symplectic pair with $p^{\prime}$, and either forms a symplectic pair with $p$ or is collinear with $p$. Moreover, there is some point $z$ collinear with all points of $M, z \in S \backslash \pi$. Then $\left\{p^{\prime}, z\right\}$ is a symplectic pair and either $\{p, z\}$ is symplectic, or $p$ is collinear with $z$. Also, each 
point of $\pi$ either form a symplectic pair with $z$ or is collinear with it. Hence if we endow the set of all points collinear or forming a symplectic pair with both $p, p^{\prime}$, then there is a maximal clique properly containing a projective plane.

This way we can distinguish pairs at distance 3 from special pairs, and symplectic pairs from collinear pairs. This concludes the proof of Corollary 6.5.

Remark 6.26 The notion of round-up triple in a spherical building seems to be intimately connected with projective embeddings of the corresponding Grassmann geometry. Indeed, whenever a polarized embedding of the latter exists for which a line of the ambient projective space carries three points of the Grassmann geometry, then the three corresponding residues form round-up triple in the building. So it might be of interest to determine all the round-up triples of spherical buildings which are not contained in a line of the corresponding Grassmann geometry. Let's call such round-up triples exceptional. Then from the proofs in the present paper, one can deduce that every (non-thin) building whose diagram has a double bond admits exceptional round-up triples (and in fact, these triples are related to central collineations with center of self-opposite type). In buildings with simply laced diagram we have found no exceptional round-up triples so far, and it is tempting to conjecture that there are no at all. Also, we mention without proof that all spherical Moufang buildings of rank 2 other than the projective planes admit exceptional round-up triples.

Concerning geometric lines, the possibilities are more limited. Indeed, it follows from Lemmas 4.8 and 4.9 and from [13] that in the following cases, we have geometric lines that are not lines in the Grassmann geometry:

(B) The building arises from a parabolic polar space and the geometric line consists of the set $S$ of maximal singular subspaces sharing a common codimension 2 subspace $X$ such that in the generalized quadrangle defined by the singular subspaces properly containing $X$, the set $S$ forms one system of lines of a maximal grid;

(C) The building arises from a symplectic polar space and the geometric line consists of a set $S$ of non-maximal singular subspaces of the same dimension $i$ containing a common subspace $X$ of dimension $i-1$ such that in the symplectic polar space defined by the singular subspaces properly containing $X$, the set $S$ is a hyperbolic line;

(F) The building arises from a metasymplectic space with the symplecta isomorphic to symplectic polar spaces and the geometric line is a geometric line of points in a symplecton.

$(\mathrm{G})$ The building arises from the split Cayley hexagon and the geometric line is either an ideal line, or the underlying field is perfect with characteristic 2 and the geometric line is an imaginary line, i.e., a non-isotropic line with respect to the symplectic polarity in projective 5 -space in which the hexagon is naturally embedded.

Note that the above cases precisely correspond to the case of "split" spherical Moufang buildings with a diagram containing a bond of even weight (i.e., in terms of algebraic 
groups, buildings with absolute and relative diagram $\mathrm{B}_{n}, n \geq 2, \mathrm{C}_{n}, n \geq 3, \mathrm{~F}_{4}$ and $\mathrm{G}_{2}$ ). It is tempting to conjecture that no other cases occur, except possibly for 'exceptional' geometric lines consisting of vertices of type 2 in buildings of relative and absolute type $\mathrm{F}_{4}$.

\section{Acknowledgments}

The first author thanks Prof. Hendrik Van Maldeghem and the University of Ghent for their generosity that made possible her visits to the University of Ghent, during which part of this work was done.

\section{References}

[1] P. Abramenko, K. Brown, Buildings: Theory and Applications, Springer, 2008.

[2] P. Abramenko, H. Van Maldeghem, Maps between buildings that preserve a given Weyl distance, Indagationes Mathematicae, 15 (2004), 305-319.

[3] R.J. Blok, A.E. Brouwer, The geometry far from a residue, Groups and Geometries (Siena 1996), 29-38, Trends Math., Birkhauser, Basel, 1998.

[4] N. Bourbaki, Groupes et Algèbres de Lie, Chapters 4,5 and 6, Actu. Sci. Ind. 1337, Hermann, paris, 1968.

[5] E. Govaert, H. Van Maldeghem, Distance-preserving maps in generalized polygons, Part II: Maps on points and/or lines, Beitr. Alg. Geom. 43 (2002), 303-324.

[6] A. Kasikova, Simple connectedness of hyperplane complements in some geometries related to buildings, J. Combin. Th. Ser. A, to appear.

[7] A. Kasikova, E.E. Shult, Point-line characterisations of Lie incidence geometries, Adv. Geom. 2 (2002), 147-188.

[8] A. Kasikova, Characterization of some subgraphs of point-collinearity graphs of building geometries, European J. Combin., 28 (2007), 1493-1529.

[9] A. Pasini, Diagram Geometries, Clarendon Press, 1994.

[10] M. Ronan, Lectures on Buildings, Academic Press, 1989.

[11] E.E. Shult, Points and Lines, Springer, 2011.

[12] J. Tits, Buildings of Spherical Type and Finite BN-Pairs, Lect. Notes Math. 386, Springer-Verlag, 1974. 
[13] J. van Bon, H. Cuypers, H. Van Maldeghem, Hyperbolic lines in generalized polygons, Forum Math. 8 (1994), 343-362.

[14] H. Van Maldeghem, Generalized Polygons, Birkhäuser, 1998.

Adresses of the authors:

Anna Kasikova

Department of Mathematics and Statistics,

Bowling Green State University,

Bowling Green, OH 43403,

USA

annakas@bgsu . edu

Hendrik Van Maldeghem

Department of Mathematics,

Ghent University,

Krijgslaan 281, S22,

9000 Gent,

BELGIUM

hvm@cage.UGent. be 